\title{
THE EFFECT OF CORIOLIS FORCE ON NONLINEAR CONVECTION IN A POROUS MEDIUM
}

\author{
D.H. RIAHI \\ Department of Theoretical and Applied Mechanics \\ University of Illinois at Urbana-Champaign \\ Urbana, Illinois 61801
}

(Received January 6, 1993)

\begin{abstract}
Nonlinear convection in a porous medium and rotating about vertical axis is studied in this paper. An upper bound to the heat flux is calculated by the method initiated first by Howard [6] for the case of infinite Prandtl number.

For $T_{a} \ll 0(1)$, the rotational effect is not significant. For $0(1) \ll T_{a} \ll 0(\sqrt{R} \log R)$, the Nusselt number decreases with increasing $T_{a}$ for a given Rayleigh number $R$. The flow has always a finite number of modes, but with increasing $T_{a}$ in this region, the number of modes decreases. The functional dependence of the Nusselt number on $R$ and $T_{a}$ is found to have discontinuities as the number of modes $N^{*}$ reduces to $N^{*}-1$. For $0(\sqrt{R} \log R) \ll T_{a} \ll 0(R)$, the Nusselt number is proportional to $\frac{R}{T_{a}}\left(\log \frac{R}{T_{a}}\right)$. The stabilizing effect of rotation is so strong that the optimal solution has left with only one horizontal mode. For $T_{a}=0(R)$, the Nusselt number becomes $0(1)$ and the convection is inhibited entirely by rotation for $T_{a}>\frac{1}{\pi^{2}} R$.
\end{abstract}

KEY WORDS AND PHRASES. Convection, rotation, nonlinear, porous medium, nonlinear convection.

1991 AMS SUBJECT CLASSIFICATION CODES. 76R10, 76R99, 76D10, 76U05, 76D30, 80A20.

\section{INTRODUCTION AND DISCUSSION.}

This is a study about nonlinear convecting fluid in a porous medium. We shall study the influence of Coriolis force on the heat transfer in turbulent convection of a porous layer of fluid using the upper bound calculation initiated first by Howard [6]. The fluid is assumed to have an infinite Darcy-Prandtl number and is bounded by two horizontal planes. As a mathematical model, we consider the Darcy-Oberbeck-Boussinesq equations (hereafter called DOB) for which a convenient reference is Lapwood [7]. The DOB equations differ from the usual Boussinesq equations for convection in an ordinary fluid layer (Chandrasekhar [4]) only as the viscous term $\nabla^{2} \underline{u}$ is replaced by $-\frac{\underline{u}}{\kappa_{1}}$, where $\kappa_{1}$ is the Darcy-permeability coefficient. The velocity $\underline{u}$ is defined according to Darcy's law as an average over the microscale of the porous medium.

Through experimental observation, we find that Darcy's law is not expected to be valid above a certain value of the seepage velocity. A universal characterization of this certain value has not yet been obtained. However, the results obtained by Gupta and Joseph [5] of an asymptotic analysis $(R \rightarrow \infty)$ using DOB equations for the non-rotating system are in qualitative agreement with the result of the well known dimensional argument relating the Nusselt number to the Rayleigh number and also with the experimental datas [5]. 
We therefore follow Busse and Joseph [1] and Gupta and Joseph [5] and assume that DOB equations hold in turbulent regime. We hope that the analysis of the present problem based on the DOB equations also gives a qualitative picture of the high Rayleigh number convection in a rotating porous medium.

Malkus [8] suggested that the actual heat transferred by the realized solution is the maximum possible. Here to produce a solvable problem, we used the strengthened form of the Malkus principle: the heat transport which actually occurs for large Rayleigh number may be almost as large as the maximum obtainable using the two so-called power integrals which are derived from the governing equations or some differential constraints which are more restrictive than these power integrals. Now regardless of the validity of the Malkus principle, the maximum heat flux subject to these power integrals or differential constraints gives an upper bound on the heat transport which actually occurs in the real world and is certainly of interest. It happened that this upper bound has some agreement with the actual heat transport and in the case of infinite Prandtl number turbulent convection (Chan [2]) has a very good agreement with the experimental observation. Thus the Malkus principle perhaps has some similarity with physical reality, and we find it appropriate to use this method for the study of the present subject. Note that we cannot expect any close relationship between the maximizing fields of velocity and temperature to those which actually occur. For example, real convection is time dependent, whereas time disappears completely from our maximizing problem. The Darcy-Prandtl number, which is very large in practice, is taken to be infinity in the present problem. The fact that the Darcy-Prandtl number is very large for natural materials means that thermally driven motion in porous material will ordinarily be very slow motion compared to the corresponding one in ordinary fluid layer.

The main purpose of studying the present problem is to develop a simple variational method to obtain at least some qualitative results from the maximizing nonlinear convecting fluid in a rotating porous medium, to compare the qualitative multi modal results of the present study with the corresponding one in ordinary convection (Chan [3]) which gave a rather unexpected behavior of the horizontal modes, and to develop a mathematical model for a possible application in fluid mechanics.

Chan [3], considered the corresponding problem in ordinary convection with free-free boundaries and found that for $T_{a} \leq 0(R)$, the flow is essentially non-rotating and there is only one single mode in the system. However, for $0(R) \leq T_{a} \leq 0\left(R^{4 / 3}\right)$, the fluid has finitely many modes, though the total number of modes increases as $T_{a}$ increases in this region, and for $0\left(R^{4 / 3}\right) \leq T_{a} \leq 0\left(R^{3 / 2}\right)$ the maximizing flow has infinitely many modes. But, heat flux decreases in $0(R) \leq T_{a} \leq 0\left(R^{3 / 2}\right)$ as $T_{a}$ increases for a given $R$. This behavior of the ordinary maximizing flow has been discussed in detail by Chan, although he also feels that if the effect of a rotational constraint is to suppress convection, the stronger the rotation, the more it will tend to suppress small eddies, and therefore the less number of modes allowed. But he noted that this contradiction to his asymptotic result can be easily resolved by saying that the number of modes is allowed to increase indefinitely in its effort to remove the rotational constraint, although the heat flux is still less than its non-rotating value. In our present problem with a porous layer of fluid, we find that for $T_{a} \ll 0(1)$, the stabilizing effect of rotation is so weak that the fluid acts as in the non-rotating system. There are essentially infinitely many modes associated with small scales of motion (or eddies). The Nusselt number increases as the Rayleigh number increases. Gupta and Joseph [5] used a less formal method to find multi-wave number solutions in non- 
rotating system and obtained the same qualitative results to the leading order terms. However. in their result for the Nusselt number, they obtained a different proportionality constant from ours. One reason for this difference is that they did not use a formal multiple-boundary layer method to solve the problem and similar to Chan [2] they obtained the Euler equations after they simplified the expression (2.11) below by using (5.10) and (5.21). For $0(1) \ll T_{a} \ll 0(R)$, the external constraint (rotation) tends to stabilize the flow. As $T_{a}$ increases in this range, the rotation tends to suppress the small eddies and therefore the number of modes decreases. The flow has always a finite number of modes. The Nusselt number drops sharply at certain values of $T_{a}$ in which the number of modes $N^{*}$ reduces to $N^{*}-1$, except for $N^{*}=2$ in which the functional dependence of the Nusselt number on $R$ and $T_{a}$ is continuous. The heat flux is always less than its non-rotating value in the entire range $0(1) \ll T_{a} \ll 0(R)$. The qualitative picture of the modes in a porous medium is therefore different from the case in which we have an ordinary fluid.

Porous medium probably was important to present this difference, although one may expect that our qualitative results should also hold in ordinary convection.

In section 2, the basic equations are derived. The instability analysis as was needed partly for the latter sections is done briefly in section 3, and finally the boundary layer analysis for a single and for many modes is done in sections 4 and 5 respectively.

\section{FORMULATION OF THE PROBLEM.}

We consider a horizontally infinite layer of fluid of depth $d$, bounded above and below. The upper and lower surfaces are maintained at temperatures $T_{0}$ and $T_{0}+\Delta T$ respectively. The fluid is rotating about the vertical with angular velocity $\Omega$. It is convenient to use nondimensional variables in which length, velocities, time, and temperature are scaled respectively by $d, \frac{K}{d}, \frac{d^{2}}{K}$, and $\triangle T$. Here $K$ is the thermal diffusivity. Then, absorbing the centrifugal force into the pressure term, and with the usual DOB equations that the density variations are taken into account only in the buoyancy term, the basic equations are:

$$
\begin{gathered}
B\left\{\frac{d u}{d t}+\underline{u} \cdot \nabla \underline{u}\right\}+\frac{2}{E}(\underline{K} \times \underline{u})=-\nabla P+R T \underline{K}-\underline{u}, \\
\nabla \cdot \underline{u}=0, \\
\frac{d T^{*}}{d t}+\underline{u} \cdot \nabla T^{*}=\nabla^{2} T^{*} .
\end{gathered}
$$

Here $\underline{u}=(u, v, w)$ is the velocity vector, $T^{*}$ is the temperaturc cuccoss 0 ver $T_{0}, T$ is the deviation of $T^{*}$ from its horizontal average $\bar{T}^{*}$, and $P$ is the deviation of the modified pressure from the hydrostatic value appropriate to $\bar{T}^{*}$, also $\underline{K}$ is the unit vector in $z$-direction, $B^{-1}=\frac{\nu d^{2}}{K K_{1}}$ is the Prandtl-Darcy number, $E=\frac{\nu}{\Omega K_{1}}$ is the Ekman number, $R=\frac{\alpha g K_{1} d \Delta T}{\nu K}$ is the Rayleigh number, where $K_{1}$ is the Darcy-permeability coefficient, $g$ is the acceleration due to gravity, and $\alpha$ is the coefficient of thermal expansion. The equations (2.1)-(2.3) differ from the Boussinesq equations for convection in an ordinary fluid layer only in so far as the term $\nabla^{2} \underline{u}$ is replaced by $-\underline{u}$. The upper bound method assumes that the flow is statistically steady in time and homogeneous in horizontal planes, and various functions describing the flow exist and are bounded.

Following previous workers, we rewrite the following power integrals:

$$
\begin{gathered}
<|\underline{u}|^{2}>=R<W T>, \\
\frac{d \bar{T}^{*}}{d z}=\overline{W T}-1-<W T>,
\end{gathered}
$$




$$
<W T>+<W T\rangle^{2}-\left\langle\overline{W T}^{2}\right\rangle=\left\langle|\nabla T|^{2}\right\rangle,
$$

which are derived from (2.1)-(2.3) [1]. Bar denotes horizontal averages, and angle brackets denote a further vertical averaging over the whole layer. It follows that the Nusselt number $N$, which measures the ratio of the actual heat transfer to that achieved simply by conduction, is

$$
N=1+<W T\rangle .
$$

Although (2.4) was used in place of (2.1) by Busse and Joseph [1], we shall not use it here, but rather follow Gupta and Joseph [5] in using (2.1) in the linear form

$$
\frac{2}{E}(\underline{K} \times \underline{u})=-\nabla P+R T \underline{K}-\underline{u},
$$

which is obtained by setting $B=0$ in (2.1). The physically appropriate value $B=0$ follows from extraordinarily small values of the permeability coefficient $K_{1}$ in porous material: in sand, $K_{1}=0\left(10^{-8}\right) \mathrm{cm}^{2} ;$ in very porous fiber metals, $K_{1}=0\left(10^{-4}\right) \mathrm{cm}^{2}$.

We shall be concerned with the problem of maximizing $N=1+\langle W T\rangle$ subject to the constraints (2.2), (2.5), and (2.7). Eliminating $P, u$, and $v$ while introducing the vertical component of vorticity $\chi=\frac{d v}{d x}-\frac{d u}{d y}$, we obtain

$$
\begin{gathered}
\nabla^{2} w-R \nabla_{1}^{2} T+\frac{2}{E} \frac{d \chi}{d z}=0 \\
-\chi+\frac{2}{E} \frac{d w}{d z}=0
\end{gathered}
$$

where $\nabla_{1}^{2}=\partial^{2} / \partial x^{2}+\partial^{2} / \partial y^{2}$. We shall scale our dependent variables again so that

$$
\left.\omega=\langle W T\rangle^{-\frac{1}{2}} R^{-\frac{1}{2}} W, \theta=\langle W T\rangle^{-\frac{1}{2}} R^{\frac{1}{2}} T, \bar{Z}=\frac{1}{2}<W T\right\rangle^{-\frac{1}{2}} R^{-\frac{1}{2}} E \chi .
$$

This converts the problem to the one of maximizing

$$
N-1=\langle W T\rangle=\frac{\left.1-R^{-1}<|\nabla \theta|^{2}\right\rangle}{\left\langle(1-\overline{\omega \theta})^{2}\right\rangle}
$$

subject to the integral constraint

$$
<\omega \theta>-1=0
$$

and the differential equation constraints

$$
\nabla^{2} \omega-\nabla_{1}^{2} \theta+T_{a} \frac{d \bar{Z}}{d z}=0, \frac{d w}{d z}=\bar{Z} \quad\left(T_{a} \equiv 4 / E^{2}\right) .
$$

Boundary conditions are

$$
\omega=\theta=\nabla^{2} \theta=0 \text { at } z=0,1 .
$$

Here $\nabla^{2} \theta=0$ arises as a natural boundary condition.

Euler equations for the maximization problem are obtained by introducing the Lagrange constant multiplier $\lambda$; multiplier functions $q^{\prime}(x, y, z)$, and $P^{\prime}(x, y, z)$, and maximizing the functional $G$ where

$$
G=N-1+2 \lambda^{\prime}<\omega \theta-1>+2<q^{\prime}\left(\nabla^{2} \omega-\nabla_{1}^{2} \theta+T_{a} \frac{d \bar{Z}}{d z}\right)+2<P^{\prime}\left(-\bar{Z}+\frac{d \omega}{d z}\right)>.
$$

The equations obtained by considering variations of $\theta, \omega$ and $\bar{Z}$ are respectively

$$
\begin{gathered}
\frac{1}{R} \nabla^{2} \theta-\nabla_{1}^{2} q+(N-1)(1-\overline{\omega \theta}) \omega+\lambda \omega=0, \\
\nabla^{2} q-\frac{d P}{d z}+(N-1)(1-\overline{\omega \theta}) \theta+\lambda \theta=0,
\end{gathered}
$$




$$
-P-T_{a} \frac{d q}{d z}=0
$$

where we defined $(\lambda, P, q)=\left\langle(1-\overline{W \theta})^{2}\right\rangle\left(\lambda^{\prime}, P^{\prime}, q^{\prime}\right)$. We thus have to solve (2.13) and (2.15)(2.17). Also it can be easily shown that $\frac{1}{2} \leq \lambda \leq 1$ and so must always be an $0(1)$ quantity. We may eliminate, $P, q$ between (2.15)-(2.17) to obtain:

We then assume $\left(\nabla_{a}^{2}+T^{2}\right)\left[\frac{1}{d z^{2}}\right)\left[\frac{1}{(N-1) R} \nabla^{2} \theta+\left(1-\overline{\omega \theta}+\frac{\lambda}{N-1}\right) \omega\right]+\left(1-\overline{\omega \theta}+\frac{\lambda}{N-1}\right) \nabla_{1}^{2} \theta=0$

$$
\omega=\sum_{n}(z) \phi_{n}(x, y), \theta=\sum_{n} \theta_{n}(z) \phi_{n}(x, y), \bar{Z}=\sum_{n} \bar{Z}_{n}(z) \phi_{n}(x, y) .
$$

where $\phi_{n}$ can be solution of the equation

$$
\nabla_{1}^{2} \phi_{n}(x, y)=-\alpha_{n}^{2} \phi_{n}(x, y)
$$

for some horizontal wave number $\alpha_{n}$. Functions with different wave numbers are naturally orthogonal, and we shall choose to make the functions orthonormal, so that

$$
\overline{\phi_{n} \phi_{m}}=\delta_{n m} .
$$

This separation of variables leads us to the system of nonlinear ordinary differential equations.

$$
\begin{gathered}
\left(\frac{d^{2}}{d z^{2}}-\alpha_{n}^{2}\right) \omega_{n}+\alpha_{n}^{2} \theta_{n}+T_{a} \frac{d \bar{Z} n}{d z}=0, \\
\frac{d \omega_{n}}{d z}=\bar{Z}_{n}, \\
{\left[\left(\frac{d^{2}}{d z^{2}}-\alpha_{n}^{2}\right)+T_{a} \frac{d^{2}}{d z^{2}}\right]\left\{\frac{1}{(N-1) R}\left(\frac{d^{2}}{d z^{2}}-\alpha_{n}^{2}\right) \theta_{n}+\left[1-\overline{\omega \theta}+\frac{\lambda}{N-1}\right] \omega_{n}\right\}} \\
-\alpha_{n}^{2}\left[1-\overline{\omega \theta}+\frac{\lambda}{N-1}\right] \theta_{n}=0 .
\end{gathered}
$$

We shall solve (2.22) - (2.24) in Sections 4 and 5 using boundary layer approximation.

\section{LINEAR ANALYSIS.}

The classical results of linear instability in an ordinary fluid (Chandrasekhar [4]) are well known. The present section is a brief extension in a porous layer. Considering an infinite horizontal layer of fluid with the governing equations (2.1) - (2.3) in which a steady adverse temperature gradient is maintained and let there be no motion in the initial stage. We assume then that the initial state be slightly perturbed. Let $\underline{u}$ denote the velocity in the perturbed state and the altered temperature be $T_{s}+\theta$, where $T_{s}$ is the initial temperature. Ignoring terms of the second and higher orders in the perturbations, we obtain:

$$
\begin{gathered}
B \frac{\partial \underline{u}}{\partial t}=-\nabla P+R \theta \underline{K}-\underline{u}+\frac{2}{E} \underline{u} X \underline{K}, \\
\frac{\partial \theta}{\partial t}=w+\nabla^{2} \theta, \\
\nabla \cdot \underline{u}=0 .
\end{gathered}
$$

By taking the curl of (3.1) and the curl of the curl of (3.1) and considering their vertical components respectively, we obtain:

$$
\begin{gathered}
B \frac{\partial \bar{Z}}{\partial t}=-\bar{Z}+\frac{2}{E} \frac{\partial \omega}{\partial z} \\
B \nabla^{2}\left(\frac{\partial \omega}{\partial t}\right)=R \nabla_{1}^{2} \theta-\nabla^{2} \omega-\frac{2 \partial \bar{Z}}{E} \partial z
\end{gathered}
$$


where $\omega$ and $\bar{Z}$ are the vertical components of velocity and vorticity respectively. To find the solutions of (3.2) and (3.4) - (3.5) with the boundary conditions

$$
\omega=\theta=\nabla^{2} \theta=0 \quad \text { at } \quad z=0,1 \text {, }
$$

we assume the functions, $\omega, \theta, \bar{Z}$ are in the form

$$
(\omega, \theta, \bar{Z})=[W(z), H(z), Z(z)] \exp \left[\imath\left(\alpha_{1} x+\alpha_{2} y\right)+\sigma t\right],
$$

where $\alpha=\sqrt{\alpha_{1}^{2}+\alpha_{2}^{2}}$ is the wave number of the disturbance and $\sigma$ is a constant. Using (3.7) in (3.2), (3.4) - (3.6), and set $\sigma=0$ (marginal state of instability), we obtain

$$
\left(D^{2}-\alpha^{2}\right) H+W=0, Z=\frac{2}{E} D W,\left(D^{2}-\alpha^{2}\right) W+\frac{2}{E} D Z+\alpha^{2} R H=0,
$$

where $D=\frac{d}{d z}$. Eliminating $H$ and $Z$, we obtain

$$
\left(D^{2}-\alpha^{2}\right)\left(D^{2}-\alpha^{2}+T_{a} D^{2}\right) W=R \alpha^{2} W .
$$

(3.9) has to be solved subject to the boundary conditions

$$
W=D^{2} W=0 \text { at } z=0,1 .
$$

The proper solution for $W$ is

$$
W=A \operatorname{Sin} n \pi z,
$$

where $n$ is an integer and $A$ is a constant. Using (3.11) in (3.9), we find the lowest value of $R$ occurs when $n=1$. Thus

$$
R=\frac{1}{\alpha^{2}}\left[\left(\pi^{2}+\alpha^{2}\right)\left(\pi^{2}+\alpha^{2}+\pi^{2} T a\right)\right] .
$$

$R$ attains its minimum $R_{c}=\pi^{2}\left(1+\sqrt{1+T_{a}}\right)^{2}$ when $\alpha=\pi^{2} \sqrt{1+T_{a}}$. As $T_{a} \rightarrow \infty$, we obtain the asymptotic forms

$$
R_{c} \rightarrow \pi^{2} T_{a}, \quad \alpha_{c} \rightarrow \pi^{2} \sqrt{T_{a}} .
$$

We now consider the case when $\sigma \neq 0$, assumed $\sigma$ is pure imaginary and seek the conditions for such solutions to exist (We assume also that $B \neq 0$ and $T_{a} \neq 0$ ). We then find from (3.2), (3.4) - (3.7) that an overstability cannot occur if

$$
\frac{3-2 \sqrt{2}}{\pi^{2} T_{a}}<B<\frac{3+2 \sqrt{2}}{\pi^{2} T_{a}} .
$$

For the particular cases $B=0$ or $T_{a}=0$, it can be easily shown that overstability never occurs.

We shall consider the nonlinear analysis in the next section. The Rayleigh number $R$ and the Nusselt number $N$ are supposed to be large and the solutions will be obtained for different ranges of $T_{a}$ in $\left(R, T_{a}\right)$ plane.

\section{SINGLE WAVE NUMBER SOLUTION.}

(a) $T_{a}=0$.

The solution for this case is of course that of Gupta and Joseph. The wave number $\alpha_{1}$ is supposed to be large, as can be justified a posterior. We have to solve the following equations and boundary conditions:

$$
\begin{gathered}
\left(\frac{d^{2}}{d z^{2}}-\alpha_{1}^{2}\right) \omega_{1}+\alpha_{1}^{2} \theta_{1}=0 \\
\frac{d \omega_{1}}{d z}=\bar{Z}_{1}, \\
\left(\frac{d^{2}}{d z^{2}}-\alpha_{1}^{2}\right)\left\{\frac{1}{(N-1) R}\left(\frac{d^{2}}{d z^{2}}-\alpha_{1}^{2}\right) \theta_{1}+\left[1-\overline{\omega \theta}+\frac{\lambda}{N-1}\right] \omega_{1}\right\}-\alpha_{1}^{2}\left[1-\overline{\omega \theta}+\frac{\lambda}{N-1}\right] \theta_{1}=0,
\end{gathered}
$$




$$
\omega_{1}=\theta_{1}=\frac{d^{2} \theta_{1}}{d z^{2}}=0 \quad \text { at } \quad z=0,1
$$

We shall obtain the solutions for $\omega_{1}, \theta_{1}$, and $\bar{Z}_{1}$ by considering three distinct regions; the interior, the intermediate layer, and the inner layer.

In the interior we assume $z$ is $0(1)$. Then (4.1) - (4.3) reduce to the following equations:

$$
\omega_{1}=\theta_{1}, \frac{d \omega_{1}}{d z}=\bar{Z}_{1}, \frac{-\alpha_{1}^{2}}{N R} \theta_{1}+\left(1-\omega_{1} \theta_{1}\right)\left(\omega_{1}+\theta_{1}\right)=0 .
$$

We then assume that $\alpha_{1}^{2} \ll N R$ as $R, N$ and $\alpha_{1}$ go to infinity. Thus, we get the following results;

$$
\omega_{1}=\theta_{1}=1, \bar{Z}_{1}=0
$$

Near each surface and adjacent to the interior, there are intermediate layers of thickness $0\left(\alpha_{1}^{-1}\right)$. Defining an appropriate variables $\xi=\alpha_{1} z$ for the lower of these layers (the upper layer is similar), the governing equations are

$$
\left(\frac{d^{2}}{d \xi^{2}}-1\right) \omega_{1}+\theta_{1}=0, \quad \omega_{1} \theta_{1}=1, \quad \bar{Z}_{1}=\alpha_{1} \frac{d \omega_{1}}{d \xi} .
$$

The governing equation for $\omega_{1}$ is

$$
\frac{d^{2} \omega_{1}}{d \xi^{2}}-\omega_{1}+\frac{1}{\omega_{1}}=0
$$

and we should require $\omega_{1} \rightarrow 1$ as $\xi \rightarrow \infty$. We also require $\omega_{1} \rightarrow 0$ as $\xi \rightarrow 0$, and the asymptotic form of $\omega_{1}$ as $\xi \rightarrow 0$ is readily found to be

$$
\omega_{1} \simeq \sqrt{2} \xi\left(\log \frac{1}{\xi}\right)^{\frac{1}{2}}
$$

and similarly

$$
\theta_{1} \simeq \frac{1}{\sqrt{2}} \xi^{-1}\left(\log \frac{1}{\xi}\right)^{-\frac{1}{2}}, \bar{Z}_{1} \simeq \sqrt{2} \alpha_{1}\left(\log \frac{1}{\xi}\right)^{\frac{1}{2}}
$$

There has to be one further layer in which $\theta_{1}$ is brought to its zero boundary value. We suppose that this inner layer is of thickness $\varepsilon \ll \alpha_{1}^{-1}$, and define as the appropriate independent variable $\eta=z / \varepsilon$. We first determine the matching conditions as $\eta \rightarrow \infty$. We define

$$
\omega_{1}=C \widehat{\omega}_{1}, \quad \theta_{1}=C^{-1} \widehat{\theta}_{1}, \bar{Z}_{1}=L \widehat{Z}_{1},
$$

where $\widehat{\omega}_{1}, \widehat{\theta}_{1}$, and $\widehat{Z}_{1}$ are order one quantities. We have also assumed that $\omega_{1} \theta_{1} \sim 0(1)$ in this region, then

which give

$$
\begin{gathered}
C \widehat{\omega}_{1}(\eta \rightarrow \infty) \simeq \sqrt{2} \xi\left(\log \frac{1}{\xi}\right)^{\frac{1}{2}}=\sqrt{2} \alpha_{1} \varepsilon \eta\left(\log \frac{1}{\alpha_{1} \varepsilon \eta}\right)^{\frac{1}{2}} \\
L \widehat{Z}_{1}(\eta \rightarrow \infty) \simeq \sqrt{2} \alpha_{1}\left(\log \frac{1}{\alpha_{1} \varepsilon \eta}\right)^{\frac{1}{2}}
\end{gathered}
$$

$$
\begin{gathered}
C=\sqrt{2} \alpha_{1} \varepsilon\left(\log \frac{1}{\alpha_{1} \varepsilon}\right)^{\frac{1}{2}}, L=\sqrt{2} \alpha_{1}\left(\log \frac{1}{\alpha_{1} \varepsilon}\right)^{\frac{1}{2}} \\
\widehat{\omega}_{1}(\eta \rightarrow \infty) \simeq \eta, \quad \widehat{\theta}_{1}(\eta \rightarrow \infty) \simeq \eta^{-1}, \quad \widehat{Z}_{1}(\eta \rightarrow \infty) \simeq 1
\end{gathered}
$$

The governing equations (3.1) - (3.3) therefore reduce to

$$
\frac{d^{2} \widehat{\omega}_{1}}{d \eta^{2}}=0, \quad \widehat{Z}_{1}=\frac{d \widehat{\omega}_{1}}{d \eta}, \quad \frac{1}{N R \varepsilon^{2} C^{2}} \frac{d^{2} \widehat{\theta}_{1}}{d \eta^{2}}=\left(\widehat{\omega}_{1} \widehat{\theta}_{1}-1\right) \widehat{\omega}_{1},
$$


which predicts that the thickness of the inner layer that must satisfy the equation

$$
2 N R \varepsilon^{4} \alpha_{1}^{2}\left(\log \frac{1}{\alpha_{1} \varepsilon}\right)=1
$$

The solutions are:

$$
\widehat{\omega}_{1}=\eta, \quad \widehat{Z}_{1}=1, \quad \widehat{\theta}_{1}=\frac{1}{2} \eta \int_{0}^{1} e^{-\frac{1}{2} \eta^{2} t}\left(1-t^{2}\right)^{-\frac{1}{4}} d t .
$$

For the constraint (2.11), we calculate the approximate expressions for

$$
\left\langle|\nabla \theta|^{2}\right\rangle=\left\langle\alpha_{1}^{2} \theta_{1}^{2}+\left(\frac{d \theta_{1}}{d z}\right)^{2}\right\rangle \simeq \alpha_{1}^{2}+\frac{3}{\varepsilon C^{2}}\left\langle\left(\frac{d \widehat{\theta}_{1}}{d \eta}\right)^{2}\right\rangle .
$$

$\left.\left\langle(1-\overline{\omega \theta})^{2}\right\rangle \sim 2 \varepsilon<\left(1-\widehat{\omega}_{1} \widehat{\theta}_{1}\right)^{2}\right\rangle$. Using these expressions, (4.12), and (4.15) in (2.11), we obtain

$$
R \simeq \alpha_{1}^{2}+\frac{I}{\alpha_{1}^{2} \varepsilon^{3}\left(\log \frac{1}{\alpha_{1} \varepsilon}\right)}, I=\int_{0}^{\infty}\left(1-\eta \widehat{\theta}_{1}\right) d \eta=2^{-\frac{1}{2}} \Gamma^{2}(3 / 4) \simeq 1.062
$$

(Gupta and Joseph [5]).

From (4.15) and (4.17), we obtain

$$
\begin{aligned}
N & \simeq \frac{1}{I}\left(\frac{1}{I}\right)^{4 / 3}\left(\frac{1}{3}\right)^{1 / 3}\left(R \alpha_{1}^{2}\right)^{1 / 3}\left(1-\alpha_{1}^{2} / R\right)^{4 / 3}\left(\log \frac{R}{\alpha_{1}}\right)^{1 / 3}, \\
\varepsilon & \simeq(3 I)^{1 / 3}\left(R \alpha_{1}^{2}\right)^{-1 / 3}\left(1-\alpha_{1}^{2} / R\right)^{-1 / 3}\left(\log \frac{R}{\alpha_{1}}\right)^{-1 / 3} .
\end{aligned}
$$

Then if $N$ is maximized with respect to $\alpha_{1}$, maximum value is

attained when

$$
N \simeq\left(\frac{2}{1}\right)^{4 / 3}\left(\frac{1}{5}\right)^{5 / 3}\left(\frac{1}{3}\right)^{1 / 3} R^{2 / 3}(\log R)^{1 / 3},
$$

\section{(b) $\quad T_{\varepsilon} \ll O(1)$.}

Rotational effects do not become significant for $T_{a} \ll 0(1)$ but can initially be regarded as small perturbations to the previous solution. Our previous solution for $\bar{Z}_{1}$ shows that the term $\frac{d \bar{Z}_{1}}{d z}$ in (2.23) first becomes significant in the intermediate layer when $T_{a}$ becomes $0(1)$. Hence rotational effects are unimportant so long as $T_{a} \ll 0(1)$.

(c) $T_{\bullet}=0(1)$

The governing equations and boundary conditions are

$$
\begin{gathered}
\left(\frac{d^{2}}{d z^{2}}-\alpha_{1}^{2}\right) \omega_{1}+\alpha_{1}^{2} \theta_{1}+T_{a} \frac{d \bar{Z}_{1}}{d z}=0 \\
\frac{d \omega_{1}}{d z}=\bar{Z}_{1}, \\
{\left[\frac{d^{2}}{d z^{2}}-\alpha_{1}^{2}+T_{a} \frac{d^{2}}{d z^{2}}\right]\left\{\frac{1}{(N-1) R}\left(\frac{d^{2}}{d z^{2}}-\alpha_{1}^{2}\right) \theta_{1}+\left(1-\omega_{1} \theta_{1}+\frac{\lambda}{N-1}\right) \omega_{1}\right\}} \\
=\alpha_{1}^{2}\left(1-\omega_{1} \theta_{1}+\frac{\lambda}{N-1}\right) \theta_{1}, \\
\omega_{1}=\theta_{1}=\frac{d^{2} \theta_{1}}{d z^{2}}=0 \text { at } z=0,1 .
\end{gathered}
$$

We shall consider the same regions and use the same notation and assumptions as in (a). In the interior (4.21) - (4.23) reduce to (4.5) and the solutions (4.6) are then obtained. 
In the intermediate layer, the governing equations become

$$
\begin{gathered}
\left(\frac{d^{2}}{d \xi^{2}}-1\right) \omega_{1}+\theta_{1}+T_{a} \frac{d^{2} \omega_{1}}{d \xi^{2}}=0 \\
\alpha_{1} \frac{d \omega_{1}}{d \xi}=\bar{Z}_{1}, \omega_{1} \theta_{1}=1 .
\end{gathered}
$$

The equation for $\omega_{1}$ is

$$
\left(1+T_{a}\right) \frac{d^{2} \omega_{1}}{d \xi^{2}}-\omega_{1}+\frac{1}{\omega_{1}}=0
$$

and we require $\omega_{1} \rightarrow 1$ as $\xi \rightarrow \infty$ and $\omega_{1} \rightarrow 0$ as $\xi \rightarrow 0$. The asymptotic form of $\omega_{1}$ as $\xi \rightarrow 0$ is

and similarly

$$
\omega_{1} \simeq \sqrt{\frac{2}{1+T_{a}}} \xi\left(\log \frac{1}{\xi}\right) \frac{1}{2}
$$

$$
\theta_{1} \simeq \sqrt{\frac{1+T_{a}}{2}} \xi^{-1}\left(\log \frac{1}{\xi}\right)^{-\frac{1}{2}}, \bar{Z}_{1} \simeq \sqrt{\frac{2}{1+T_{a}}} \alpha_{1}\left(\log \frac{1}{\xi}\right)^{\frac{1}{2}}
$$

In the inner layer we obtain easily the following results

$$
C=\sqrt{\frac{2}{1+T_{a}}} \alpha_{1} \varepsilon\left(\log \frac{1}{\alpha_{1} \varepsilon}\right)^{\frac{1}{2}}, \quad L=\sqrt{\frac{2}{1+T_{a}}} \alpha_{1}\left(\log \frac{1}{\alpha_{1} \varepsilon}\right)^{\frac{1}{2}}
$$

and (4.13), (4.14), and (4.16) are obtained easily. Clearly the expressions for $\varepsilon$ and $N$ are

$$
\begin{gathered}
N \simeq\left(1+T_{a}\right)^{-1 / 3}\left(\frac{1}{2}\right)\left(\frac{1}{3}\right)^{1 / 3}\left(R \alpha_{1}^{2}\right)^{1 / 3}\left(1-\alpha_{1}^{2} / R\right)^{4 / 3}\left(\frac{1}{I}\right)^{4 / 3}\left(\log \frac{R}{\alpha_{1}}\right)^{1 / 3} \\
\varepsilon \simeq(3 I)^{1 / 3}\left(R \alpha_{1}^{2}\right)^{-1 / 3}\left(1-\alpha_{1}^{2} / R\right)^{-1 / 3}\left(1+T_{a}\right)^{1 / 3}\left(\log \frac{R}{\alpha_{1}}\right)^{-1 / 3}
\end{gathered}
$$

Then if $N$ is maximized with respect to $\alpha_{1}$, the maximum value is

attained when

$$
N \simeq\left(\frac{2}{I}\right)^{4 / 3}\left(\frac{1}{5}\right)^{5 / 3}\left(\frac{1}{3}\right)^{1 / 3}\left(\frac{1}{1+T_{a}}\right)^{1 / 3} R^{2 / 3}(\log R)^{1 / 3},
$$

$$
\alpha_{1}=(R / 5)^{1 / 2}
$$

\section{(d) $0(1) \ll T_{a} \ll 0[\sqrt{R} \log R]$}

The governing equations are (4.21) - (4.23) with boundary conditions (4.24). We shall obtain the solutions for $\omega_{1}, \theta_{1}$, and $\bar{Z}_{1}$ by considering three distinct regions: the interior, the intermediate layer, and the inner layer. In the interior, we assume $z$ is $0(1)$. Then (4.21) - (4.23) reduce to the following equations

$$
\begin{gathered}
-\omega_{1}+\theta_{1} \frac{T_{a}}{\alpha_{1}^{2}}+\frac{d \bar{Z}_{1}}{d z}=0 \\
\frac{d \omega_{1}}{d z}=\bar{Z}_{1}, \frac{-\alpha_{1}^{2}}{(N-1) R} \theta_{1}=\left(\omega_{1} \theta_{1}-1\right)\left(\omega_{1}+\theta_{1}\right)
\end{gathered}
$$

We define$$
\omega_{1}=A \widetilde{\omega}_{1}^{-}, \theta_{1}^{-}=\frac{1}{A} \tilde{\theta}_{1}^{\sim}, \bar{Z}_{1}^{=}=K \check{Z}_{1}^{\simeq}
$$

where $\widetilde{\omega}_{1}, \widetilde{\theta}_{1}$, and $\widetilde{Z}_{1}$ are order one quantities. We have also assumed that $\omega_{1} \theta_{1} \sim 0(1)$ in all the regions, $\alpha_{1}^{2} \ll N R$, and $0(1) \ll T_{a} \ll 0\left(\alpha_{1}^{2}\right)$. Then (4.35) - (4.36) reduce to

$$
\widetilde{\omega}_{1}=\widetilde{\theta}_{1}, \widetilde{\omega}_{1} \widetilde{\theta}_{1}=1, \frac{d \widetilde{\omega}_{1}}{d z}=\widetilde{Z}_{1}, A=K=1
$$

Thus, we get the following results: 


$$
\widetilde{\omega}_{1}=\widetilde{\theta}_{1}=1, \widetilde{Z}_{1}=0
$$

In the intermediate layer of thickness $\delta_{1}=\sqrt{\frac{T_{a}}{\alpha_{1}}}$, we define the layer variable to be $\xi$ where

$$
\xi=z / \delta_{1}, \frac{1}{\alpha_{1}} \ll \delta_{1} \ll 1,
$$

and define

$$
\omega_{1}=B \dot{\omega}_{1}, \theta_{1}=\frac{1}{B} \stackrel{\circ}{1}_{1}, \bar{Z}_{1}=K_{1} \stackrel{\circ}{Z}_{1},
$$

where $\stackrel{\circ}{\omega}_{1}, \stackrel{\circ}{\theta}_{1}$, and $\stackrel{\check{Z}}{1}_{1}$ are order one quantities, then

$$
B \stackrel{\circ}{\omega}_{1}(\xi \rightarrow \infty)=1, K_{1} \stackrel{\circ}{1}_{1}(\xi \rightarrow \infty)=0 .
$$

The governing equations reduce to

$$
-\omega_{1}+\theta_{1}+\frac{d \stackrel{\circ}{1}_{1}}{d \xi}=0, \frac{d \stackrel{\circ}{\omega}_{1}}{d \xi}=\stackrel{\circ}{Z}_{1}, \stackrel{\circ}{\omega}_{1} \grave{\theta}_{1}=1
$$

We also obtain

The asymptotic solutions as $\xi \rightarrow \infty$ are

$$
B=1, K_{1}=\frac{\alpha_{1}}{\sqrt{T_{a}}}
$$

$$
\stackrel{\circ}{\omega}_{1} \simeq \sqrt{2} \xi\left(\log \frac{1}{\xi}\right) \frac{1}{2}, \stackrel{\circ}{\theta}_{1}=\stackrel{\circ}{\omega}_{1}^{-1}, \stackrel{\circ}{Z}_{1} \simeq \sqrt{2}\left(\log \frac{1}{\xi}\right)^{\frac{1}{2}}
$$

In the inner layer of thickness $\varepsilon \ll \alpha_{1}^{-1}$, we define the appropriate variable layer as $\eta=z / \varepsilon$. We first determine the matching conditions as $\eta \rightarrow \infty$. We define

$$
\omega_{1}=C \widehat{\omega}_{1}, \theta_{1}=\frac{1}{C} \widehat{\theta}_{1}, \bar{Z}_{1}=L \widehat{Z}_{1},
$$

where $\widehat{\omega}_{1}, \widehat{\theta}_{1}$ and $\widehat{Z}_{1}$ are order on quantities. Then

which give

$$
\begin{aligned}
& C \widehat{\omega}_{1}(\eta \rightarrow \infty) \simeq \sqrt{2} \xi\left(\log \frac{1}{\xi}\right)^{\frac{1}{2}}=\sqrt{2} \frac{\alpha_{1} \varepsilon}{\sqrt{T_{a}} \eta\left(\log \frac{\sqrt{T_{a}}}{\alpha_{1} \varepsilon \eta}\right)^{\frac{1}{2}},} \\
& L \widehat{Z}_{1}(\eta \rightarrow \infty) \simeq \sqrt{2}\left(\log \frac{1}{\xi}\right)^{\frac{1}{2}} \cdot \frac{\alpha_{1}}{\sqrt{T_{a}}}=\frac{\sqrt{2} \alpha_{1}}{\sqrt{T_{a}}}\left(\log \frac{\sqrt{T_{a}}}{\alpha_{1} \varepsilon \eta}\right)^{\frac{1}{2}},
\end{aligned}
$$

$$
\begin{gathered}
C=\sqrt{\frac{2}{T_{a}}} \alpha_{1} \varepsilon\left(\log \frac{\sqrt{T_{a}}}{\alpha_{1} \varepsilon}\right)^{\frac{1}{2}}, L=\sqrt{\frac{2}{T_{a}}} \alpha_{1}\left(\log \frac{\sqrt{T_{a}}}{\alpha_{1} \varepsilon}\right)^{\frac{1}{2}}, \\
\widehat{\omega}_{1}(\eta \rightarrow \infty) \simeq \eta, \widehat{\theta}_{1}(\eta \rightarrow \infty) \simeq \frac{1}{\eta}, \widehat{Z}_{1}(n \rightarrow \infty) \simeq 1 .
\end{gathered}
$$

The governing equations $(4.21)$ - (4.32) therefore reduce to

$$
\frac{d \widehat{Z}_{1}}{d \eta}=0, \frac{d \widehat{\omega}_{1}}{d \eta}=\widehat{Z}, \frac{1}{N R C^{2} \varepsilon^{2}} \frac{d^{2} \widehat{\theta}_{1}}{d \eta^{2}}=\left(\widehat{\omega}_{1} \widehat{\theta}_{1}-1\right) \widehat{\omega}_{1},
$$

which predicts that the thickness of the inner layer must satisfy the equation

$$
2 N R \alpha_{1}^{2} \varepsilon^{4}\left(\log \frac{\sqrt{T_{a}}}{\alpha_{1} \varepsilon}\right)=T_{a} .
$$

The solutions are the same as the inner layer solutions in previous cases. For the constraint (2.11), we calculate the approximate expressions for

$$
\left.\left\langle|\nabla \theta|^{2}\right\rangle=\left\langle\alpha_{1}^{2} \theta_{1}^{2}+\left(\frac{d \theta_{1}}{d z}\right)^{2}\right\rangle \simeq \alpha_{1}^{2}+\frac{2}{\varepsilon C^{2}}<\left(\frac{d \widehat{\theta}_{1}}{d \eta}\right)^{2}\right\rangle,
$$

$\left.\left\langle(1-\overline{\omega \theta})^{2}\right\rangle \sim 2 \varepsilon<\left(1-\widehat{\omega}_{1} \widehat{\theta}_{1}\right)^{2}\right\rangle$. Using these expressions, (4.47), and (4.50) into (2.11), we 
obtain

$$
R \simeq \alpha_{1}^{2}+\frac{I T_{a}}{\varepsilon^{3} \alpha_{1}^{2}\left(\log \frac{\sqrt{T_{a}}}{\alpha_{1} \varepsilon}\right)}
$$

where $I$ is defined in (4.17). From (4.50) and (4.51), we obtain

$$
\begin{gathered}
\varepsilon \simeq\left(\frac{6 I T_{a}}{R \alpha_{1}^{2}}\right)^{1 / 3}\left(1-\frac{\alpha_{1}^{2}}{R}\right)^{-1 / 3}\left(\log \frac{T_{a} R^{2}}{\alpha_{1}^{2}}\right)^{-1 / 3} \\
N \simeq(2 I)^{-4 / 3}\left(\frac{1}{3}\right)^{1 / 3}\left(\frac{R \alpha_{1}^{2}}{T_{a}}\right)^{1 / 3}\left(1-\frac{\alpha_{1}^{2}}{R}\right)^{4 / 3}\left(\log \frac{T_{a} R^{2}}{\alpha_{1}^{2}}\right)^{1 / 3}
\end{gathered}
$$

The maximizing value of $N$ is attained when

$$
\begin{aligned}
N & \simeq(2 I)^{-4 / 3}\left(\frac{1}{3}\right)^{1 / 3}\left(\frac{R^{2}}{T_{a}}\right)^{1 / 3}\left(\frac{1}{5}\right)^{5 / 3}(4)^{4 / 3}(\log R)^{1 / 3}, \\
\alpha_{1} & =(R / 5)^{\frac{1}{2}} .
\end{aligned}
$$

(4.54), our assumptions $\varepsilon \ll \frac{1}{\alpha_{1}}$, and $0(1) \ll T_{a} \ll 0\left(\alpha_{1}^{2}\right)$ give

$$
0(1) \ll T_{a} \ll 0(\sqrt{R} \log R) .
$$

The Nusselt number has the same form as (4.33) in both ranges $T_{a} \ll 0(1)$ and (4.56). We note also that rotation acts to decrease $N$ over its non-rotating value for fixed and large values of $R$.

(e) $0(\sqrt{R} \log R) \ll T_{a} \ll 0(R)$.

The governing equations are (4.21) - (4.23) with boundary conditions (4.24).

We shall obtain the solutions for $\omega_{1}, \theta_{1}$, and $\bar{Z}_{1}$ by considering two distinct regions, the interior and the inner layer.

In the interior we expect $\omega_{1} \theta_{1}=1$. Then (4.21) - (4.23) reduce to the following equations:

$$
\begin{gathered}
\left(K \frac{d^{2}}{d z^{2}}-1\right) \omega_{1}+\theta_{1}=0, \frac{d \omega_{1}}{d z}=\bar{Z}_{1}, \\
\omega_{1} \theta_{1}=1,
\end{gathered}
$$

Where we defined $K=\left(1+T_{a}\right) / \alpha_{1}^{2}$.

Thus we get the following asymptotic results near $z=0$ :

$$
\omega_{1} \simeq \sqrt{\frac{2}{K}} z\left(\log \frac{1}{z}\right)^{\frac{1}{2}}, \bar{Z}_{1} \simeq \sqrt{\frac{2}{K}}\left(\log \frac{1}{z}\right)^{\frac{1}{2}}, \theta_{1} \simeq \frac{1}{\omega_{1}}
$$

There has to be an inner layer in which $\theta_{1}$ is brought to its zero boundary value. We suppose that this inner layer is of thickness $\alpha_{1}^{-1} \ll \varepsilon \ll 1$, and define as the appropriate independent variable $z_{1}=z / \varepsilon$. We first determine the matching conditions as $z_{1} \rightarrow \infty$. We define

$$
\omega_{1}=C \widehat{\omega}_{1}, \theta_{1}=\frac{1}{C} \widehat{\theta}_{1}, \bar{Z}_{1}=L \widehat{Z}_{1},
$$

where $\widehat{\omega}_{1}, \widehat{\theta}_{1}$ and $\widehat{Z}_{1}$ are order one quantities. We have also assumed that $\omega_{1} \theta_{1} \sim 0(1)$ in this region, then

which give

$$
\begin{gathered}
C \widehat{\omega}_{1}\left(z_{1} \rightarrow \infty\right) \simeq \sqrt{\frac{2}{K}} \varepsilon z_{1}\left(\log \frac{1}{\varepsilon z_{1}}\right)^{\frac{1}{2}} \\
L \widehat{Z}_{1}\left(z_{1} \rightarrow \infty\right) \simeq \sqrt{\frac{2}{K}}\left(\log \frac{1}{\varepsilon z_{1}}\right)^{\frac{1}{2}}
\end{gathered}
$$




$$
\begin{gathered}
C=\sqrt{\frac{2}{K^{\prime}} \varepsilon\left(\log \frac{1}{\varepsilon}\right)^{\frac{1}{2}}, L}=\sqrt{\frac{2}{K}}\left(\log \frac{1}{\varepsilon}\right)^{\frac{1}{2}}, \\
\widehat{\omega}_{1}\left(z_{1} \rightarrow \infty\right) \simeq z_{1}, \widehat{\theta}_{1}\left(z_{1} \rightarrow \infty\right) \simeq \frac{1}{z_{1}}, \widehat{Z}_{1}\left(z_{1} \rightarrow \infty\right) \simeq 1 .
\end{gathered}
$$

The governing equations (4.21) - (4.23) therefore reduce to

$$
\widehat{Z}_{1}=\frac{d \widehat{\omega}_{1}}{d z_{1}}, \frac{T_{a}}{\alpha_{1}^{2} \varepsilon^{2}} \frac{d^{2} \widehat{\omega}_{1}}{d z_{1}^{2}}=\widehat{\omega}_{1}, \frac{-\alpha_{1}^{2}}{N R C^{2}} \widehat{\theta}_{1}+\left(1-\widehat{\omega}_{1} \widehat{\theta}_{1}\right) \widehat{\omega}_{1}=0,
$$

which predicts that the thickness of the inner layer must satisfy the equation

$$
2 N R \varepsilon^{2}\left(\log \frac{1}{\varepsilon}\right)=K \alpha_{1}^{2}
$$

Assuming $\alpha_{1} \varepsilon \ll \sqrt{T_{a}}$, we obtain

$$
\widehat{\theta}_{1}=z_{1} /\left(1+z_{1}^{2}\right)
$$

We now calculate the appropriate expressions

where

$$
\begin{gathered}
<|\nabla \theta|^{2}>\simeq \frac{2 \alpha_{1}^{2} \varepsilon}{C^{2}} \int_{0}^{\infty} \widehat{\theta}_{1}^{2} d z_{21}, \\
<(1-\overline{\omega \theta})^{2}>\simeq 2 \varepsilon \int_{0}^{\infty}\left(1-\widehat{\omega}_{1} \widehat{\theta}_{1}\right)^{2} d z_{1} . \\
\int_{0}^{\infty} \widehat{\theta}_{1}^{2} d z_{1}=\frac{\pi}{4}, \int_{0}^{\infty}\left(1-\widehat{\omega}_{1} \widehat{\theta}_{1}\right)^{2} d z_{1}=\frac{\pi}{4} .
\end{gathered}
$$

Using (4.68) - (4.70) in (2.11) and with (4.65), we obtain

$$
\begin{gathered}
\varepsilon \simeq \frac{\pi}{2} \frac{T_{a}}{R}\left(\log \frac{R}{T_{a}}\right)^{-1} . \\
N \simeq \frac{2}{\pi^{2}} \frac{R}{T_{a}}\left(\log \frac{R}{T_{a}}\right) .
\end{gathered}
$$

The expressions for $N$ and $\varepsilon$ are both independent of the wave number $\alpha_{1}$. Now from our previous assumptions $\varepsilon \ll 1, \alpha_{1} \varepsilon \ll 1$, and (4.66), we obtain

$$
\frac{R}{T_{a}} \log \left(\frac{R}{T_{a}}\right) \ll \alpha_{1} \ll \frac{R}{\sqrt{T_{a}}} \log \left(\frac{R}{T_{a}}\right), T_{a} \ll 0(R)
$$

The range of the validity of the solutions for this case is limited to

$$
0(\sqrt{R} \log R) \ll T_{a} \ll 0(R) .
$$

The functional dependence of the Nusselt number on $R$ and $T_{a}$ is continuous, and is equal to $\sqrt{R}$ as $T_{a} \rightarrow 0(\sqrt{R} \log R)$ in both ranges (4.56) and (4.73). As $T_{a} \rightarrow 0(R)$, the Nusselt number is no longer large and it approaches value one. However, by the result obtained in Section 3, the fluid is stable for $R \leq \pi^{2} T_{a}$.

\section{MULTI WAVES SOLUTIONS:}

a. Rotational effects are unimportant $\left[T_{a} \ll 0(1)\right]$.

The boundary layer structure for this case is essentially that of the non-rotating case (Gupta and Joseph [5]) to the leading order terms.

We shall consider three regions for each mode; the interior, the intermediate layer, and the inner layer. We coincide the interior of the $\alpha_{n}$ mode with the extent of the inner layer of the $\alpha_{n-1}$ mode. The coupling among the different modes occurs only between the $n$th and the $(n-1)$ th mode and only in the $(n-1)$ th mode. We define $\frac{1}{a_{n}}$ as the thickness of the intermediate 
layer in the $n$th mode and $\delta_{n}$ as the thickness of the inner layer in the same mode. We also define

and assume

$$
\delta_{0} \equiv 1,
$$

$$
\alpha_{n} \rightarrow \infty, \delta_{n-1} \ll \frac{1}{\alpha_{n-1}} \ll \delta_{n-2} \text { as } R \rightarrow \infty .
$$

In the interior of the $\alpha_{n}$ mode, we define

$$
\omega_{n}=A_{n} \widetilde{\omega}_{n}\left(\zeta_{n-1}\right), \theta_{n}=\frac{1}{A_{n}} \widetilde{\theta}_{n}\left(\zeta_{n-1}\right), \bar{Z}_{n}=K_{n} \widetilde{Z}_{n}\left(\zeta_{n-1}\right),
$$

where $\widetilde{\omega}_{n}, \widetilde{\theta}_{n}, \widetilde{z}_{n}$ are order one quantities and $\zeta_{n-1}=\frac{z}{\delta_{n-1}}$ is the boundary layer variable for the $(n-1)$ th mode. The basic equations $(2.22)-(2.23)$ then become

which suggest that

$$
\begin{gathered}
-\widetilde{\omega}_{n}+\frac{1}{A_{n}^{2}} \widetilde{\theta}_{n}+\frac{T_{a} K_{n}}{A_{n} \alpha_{n}^{2} \delta_{n-1}} \frac{d \widetilde{Z}_{n}}{d \zeta_{n-1}}=0, \\
A_{n} \frac{d \widetilde{\omega}_{n}}{d \zeta_{n-1}}=\delta_{n-1} K_{n} \widetilde{Z}_{n} \\
K_{n} \delta_{n-1}=A_{n}, \\
-\widetilde{\omega}_{n}+\frac{1}{A_{n}^{2}} \widetilde{\theta}_{n}+\frac{T_{a}}{\alpha_{n}^{2} \delta_{n-1}^{2}} \frac{d \widetilde{Z}_{n}}{d \zeta_{n-1}}=0 .
\end{gathered}
$$

Assuming

$$
T_{a} \ll 0(1)
$$

and using (5.2), we obtain

$$
T_{a} \ll \alpha_{n}^{2} \delta_{n-1}^{2},
$$

and so (5.7) gives

$$
A_{n}=1, \widetilde{\omega}_{n}=\widetilde{\theta}_{n} .
$$

In the intermediate layer of the $\alpha_{n}$ mode, we define

$$
\omega_{n}=B_{n} \stackrel{\circ}{\omega}_{n}\left(\xi_{n}\right), \quad \theta_{n}=\frac{1}{B_{n}} \stackrel{\circ}{n}_{n}\left(\xi_{n}\right), \quad \bar{Z}_{n}=L_{n} \stackrel{\circ}{Z}_{n}\left(\xi_{n}\right),
$$

where $\stackrel{\circ}{\omega}_{n}, \stackrel{\circ}{n}_{n}, \stackrel{\varkappa}{n}_{n}$ are order one quantities and $\xi_{n}=\alpha_{n} z$ is the intermediate layer variable for the $n$th mode. Since there is no coupling between the modes in this layer; the equation (2.24) gives

provided we assume

$$
1-\stackrel{\circ}{\omega}_{n} \stackrel{\circ}{n}_{n}=0,
$$

$$
\alpha_{n}^{2} \ll N R B_{n}^{2}
$$

The equations $(2.22)-(2.23)$ in this layer become

$$
\begin{gathered}
\left(\frac{d^{2}}{d \xi_{n}^{2}}-1\right) \stackrel{\circ}{\omega}_{n}+\frac{1}{B_{n}^{2}} \stackrel{\circ}{n}_{n}+\frac{T_{a} L_{n}}{\alpha_{n} B_{n}} \frac{d \check{Z}_{n}}{d \xi_{n}}=0, \\
\alpha_{n} B_{n} \frac{d \omega_{n}}{d \xi_{n}}=L_{n} \stackrel{\circ}{n}_{n},
\end{gathered}
$$

which suggest that

$$
L_{n}=\alpha_{n} B_{n}
$$

Using (5.8) and (5.16) in (5.14) - (5.15), we obtain

$$
\left(\frac{d^{2}}{d \xi_{n}^{2}}-1\right) \stackrel{\circ}{\omega}_{n}+\stackrel{\circ}{\theta}_{n}=0,
$$




$$
\begin{aligned}
\frac{d \stackrel{\leftrightarrow}{\omega}_{n}}{d \xi_{n}} & =\stackrel{Z}{n}_{n} \\
B_{n} & =1 .
\end{aligned}
$$

The form of the solutions to (5.12), (5.17) - (5.18) as $\xi_{n} \rightarrow 0$ can be found, after imposing the requirement that $\omega_{n} \rightarrow 0$, to be

$$
\stackrel{\circ}{\omega}_{n} \simeq \sqrt{2} \xi_{n}\left(\log \frac{1}{\xi_{n}}\right)^{\frac{1}{2}}, \stackrel{\circ}{n}_{n}=\frac{1}{\dot{\omega}_{n}}, \stackrel{\check{Z}}{n}_{n} \simeq \sqrt{2}\left(\log \frac{1}{\xi_{n}}\right)^{\frac{1}{2}}
$$

An expression for $1-\sum_{n} \omega_{n} \theta_{n}$ in various regions is needed for discussing the inner layer. It is

$$
1-\sum_{n} \omega_{n} \theta_{n}=\left\{\begin{array}{l}
0 \text { for } z \simeq 0\left(\alpha_{n}^{-1}\right) \\
1-\widehat{\omega}_{n}\left(\zeta_{n}\right) \widehat{\theta}_{n}\left(\zeta_{n}\right)-\widetilde{\omega}_{n+1}\left(\zeta_{n}\right) \tilde{\theta}_{n+1}\left(\zeta_{n}\right) \text { for } z \simeq 0\left(\delta_{n}\right) .
\end{array}\right.
$$

In the inner layer of the $\alpha_{n}$ mode, we define

$$
\omega_{n}=C_{n} \widehat{\omega}_{n}\left(\zeta_{n}\right), \quad \theta_{n}=\frac{1}{C_{n}} \widehat{\theta}_{n}\left(\zeta_{n}\right), \bar{Z}_{n}=M_{n} \widehat{Z}_{n}\left(\zeta_{n}\right)
$$

where $\widetilde{\omega}_{n}, \widetilde{\theta}_{n}, \widetilde{Z}_{n}$ are order one quantities and $\zeta_{n}=\frac{z}{\delta_{n}}$ is the inner layer variable of the $n$th mode. Matching conditions are

which give

$$
\begin{gathered}
C_{n} \widehat{\omega}_{n}\left(\zeta_{n} \rightarrow \infty\right) \simeq \sqrt{2} \alpha_{n} \delta_{n} \zeta_{n}\left(\log \frac{1}{\alpha_{n} \delta_{n} \zeta_{n}}\right)^{\frac{1}{2}} \\
\frac{1}{C_{n}} \widehat{\theta}_{n}\left(\zeta_{n} \rightarrow \infty\right) \simeq \frac{1}{\sqrt{2}} \frac{1}{\alpha_{n} \delta_{n} \zeta_{n}}\left(\log \frac{1}{\alpha_{n} \delta_{n} \zeta_{n}}\right)^{-\frac{1}{2}}, \\
M_{n} \widehat{Z}_{n}\left(\zeta_{n} \rightarrow \infty\right) \simeq \sqrt{2} \alpha_{n}\left(\log \frac{1}{\alpha_{n} \delta_{n} \zeta_{n}}\right)^{\frac{1}{2}},
\end{gathered}
$$

$$
\begin{gathered}
C_{n}=\sqrt{2} \alpha_{n} \delta_{n}\left(\log \frac{1}{\alpha_{n} \delta_{n}}\right)^{\frac{1}{2}} \\
M_{n}=\sqrt{2} \alpha_{n}\left(\log \frac{1}{\alpha_{n} \delta_{n}}\right)^{\frac{1}{2}} \\
\widehat{\omega}_{n}\left(\zeta_{n} \rightarrow \infty\right) \simeq \zeta_{n}, \widehat{\theta}_{n}\left(\zeta_{n} \rightarrow \infty\right) \simeq \frac{1}{\zeta_{n}}, \widehat{Z}_{n}\left(\zeta_{n} \rightarrow \infty\right) \simeq 1 .
\end{gathered}
$$

The governing equations (2.22) - (2.24) then become

$$
\begin{gathered}
\frac{d^{2} \widehat{\omega}_{n}}{d \zeta_{n}^{2}}=0, \widehat{Z}_{n}=\frac{d \widehat{\omega}_{n}}{d \zeta_{n}}, \\
\frac{1}{\widehat{\omega}_{n}} \frac{d^{2} \widehat{\theta}_{n}}{d \zeta_{n}^{2}}+2 N R \alpha_{n}^{2} \delta_{n}^{4}\left(\log \frac{1}{\alpha_{n} \delta_{n}}\right)\left(1-\widehat{\omega}_{n} \widehat{\theta}_{n}-\widetilde{\omega}_{n+1} \widetilde{\theta}_{n+1}\right)=0 .
\end{gathered}
$$

In this layer, we should also consider the functions $\widetilde{\omega}_{n+1}, \widetilde{\theta}_{n+1}$ and we have

$$
\begin{gathered}
\frac{-\alpha_{n+1}^{2}}{N R} \widetilde{\theta}_{n+1}+\left(1-\widehat{\omega}_{n} \widehat{\theta}_{n}-\widetilde{\omega}_{n+1} \widetilde{\theta}_{n+1}\right) \widetilde{\omega}_{n+1}=0 \\
\frac{d \widetilde{\omega}_{n+1}}{d \zeta_{n}}=\widetilde{Z}_{n+1}, \widetilde{\omega}_{n}=\widetilde{\theta}_{n} .
\end{gathered}
$$

Assuming the total number of the horizontal wave numbers is $N^{*}$, then for $n \neq N^{*}, \widetilde{\theta}_{n+1} \neq 0$, and $(5.24 b)-(5.25 a)$ give

$$
\frac{d^{2} \widehat{\theta}_{n}}{d \zeta_{n}^{2}}+2 \alpha_{n}^{2} \alpha_{n+1}^{2} \delta_{n}^{4}\left(\log \frac{1}{\alpha_{n} \delta_{n}}\right) \widehat{\omega}_{n}=0
$$


which suggest that

$$
2 \alpha_{n}^{2} \alpha_{n+1}^{2} \delta_{n}^{4}\left(\log \frac{1}{\alpha_{n} \delta_{n}}\right)=a,
$$

where $a=0(1)$ is a constant, and will be determined later. Thus (5.26) reduces to

satisfying the boundary condition

$$
\frac{d^{2} \widehat{\theta}_{n}}{d \zeta_{n}^{2}}+a \widehat{\omega}_{n}=0
$$

$$
\widehat{\theta}_{n}(0)=0 .
$$

For $n \neq N^{*}$ then as $\widetilde{\theta}_{n+1}$ decreases to zero, $\widetilde{\theta}_{n}$ must merge with $1-\widehat{\omega}_{n} \widehat{\theta}_{n}=0$ which is the leading order term of $(5.24 \mathrm{~b})$.

Now (5.24a) gives

$$
\widehat{\omega}_{n}=\zeta_{n}, \widehat{Z}_{n}=1 \quad n=1, \cdots, N^{*} .
$$

Using the transformation $\widehat{\theta}_{n}=a^{1 / 4} g, \zeta_{n}=a^{-1 / 4} \eta$ and (5.29) in (5.28), we obtain

$g(0)=0$, and merging with $g(\eta)=\frac{1}{\eta}$.

$$
\frac{d^{2} g}{d \eta^{2}}+\eta=0
$$

For $n=N^{*}, \widetilde{\theta}_{n+1} \equiv 0$ and $(5.25 \mathrm{a}, \mathrm{b})$ always satisfied, (5.24) then suggests that

$$
2 N R \delta_{N^{*}}^{4} \alpha_{N^{*}}^{2}\left(\log \frac{1}{\alpha_{N^{*}} \delta_{N^{*}}}\right)=a_{1},
$$

where $a_{1}=0(1)$ is a constant, and will be determined later. Thus (5.24b) reduces to

satisfying the boundary conditions

$$
\frac{d^{2} \widehat{\theta}_{N^{*}}}{d \zeta_{N^{*}}^{2}}+a_{1}\left(1-\zeta_{N^{*}} \widehat{\theta}_{N^{*}}\right) \zeta_{N^{*}}=0
$$

$$
\widehat{\theta}_{N^{*}}(0)=0, \widehat{\theta}_{N^{*}}\left(\zeta_{N^{*}} \rightarrow \infty\right)=\frac{1}{\zeta_{N^{*}}}
$$

Using the transformation $\zeta_{N^{*}}=a_{1 \eta}^{-1 / 4}$ and $\widehat{\theta}_{N^{*}}=a_{1}^{1 / 4} f$ in (5.34) - (5.35), we obtain

$$
\frac{d^{2} f}{d \eta^{2}}+(1-\eta f) \eta=0
$$

$f(0)=0$ and $f(\infty) \simeq \frac{1}{\eta}$.

The solution to $(5.36)$ is

$$
f(\eta)=\frac{1}{2} \eta \int_{0}^{1} e^{-\frac{1}{2} \eta^{2} t}\left(1-t^{2}\right)^{-\frac{1}{4}} d t
$$

To evaluate the expression (2.11), we write the expression $\left\langle 1 \nabla \theta 1^{2}\right\rangle$ and $\left\langle(1-\overline{\omega \theta})^{2}\right\rangle$ to the leading order terms as:

where we define

$$
\begin{gathered}
<|\nabla \theta|^{2}>\simeq \sum_{1}^{N^{*}}\left[2 \alpha_{n}^{2} \cdot \frac{g_{n-1}}{\alpha_{n-1}}<\widetilde{\theta}_{n}^{2}>+\frac{\alpha_{n}<\widehat{\theta}_{n}^{\prime 2}>}{g_{n}^{3}\left(\log \frac{1}{g_{n}}\right)}\right], \\
<(1-\overline{\omega \theta})^{2}>\simeq 2 \sum_{1}^{N^{*}} \frac{g_{n}}{\alpha_{n}}<\left(1-\zeta_{n} \widehat{\theta}_{n}-\widetilde{\theta}_{n+1}^{2}\right)^{2}>
\end{gathered}
$$

$$
\left.\delta_{n}=g_{n} / \alpha_{n},<\widetilde{\theta}_{n}^{2}\right\rangle=\frac{1}{2}, \widetilde{\theta}_{N^{*}+1} \equiv 0
$$


Using these expressions in (2.11), we obtain

$$
N \simeq \frac{1-\frac{1}{R} \sum_{1}^{N^{*}}\left[2 \alpha_{n}^{2} \frac{g_{n-1}}{\alpha_{n-1}}<\widetilde{\theta}_{n}^{2}>+\frac{\alpha_{n}<\widehat{\theta}_{n}^{\prime 2}>}{g_{n}^{3}\left(\log 1 / g_{n}\right)}\right]}{2 \sum_{1}^{N^{*}} \frac{\alpha_{N^{*}}}{g_{N^{*}}} \cdot \frac{g_{n}}{\alpha_{n}}<\left(1-\zeta_{n} \widehat{\theta}_{n}-\widetilde{\theta}_{n+1}^{2}\right)^{2}>} \cdot \frac{\alpha_{N^{*}}}{\bar{g}_{N^{*}}}
$$

To maximize $N$ with respect to the wave numbers $\alpha_{n}$, we must first determine $\alpha_{n}$ and $g_{n}$ and in order to determine them, it is appropriate to write

$$
\left\langle|\nabla \theta|^{2}\right\rangle \simeq 0(R)
$$

Hence

$$
\alpha_{n}^{2} g_{n-1} \simeq R \alpha_{n-1}, \alpha_{n} \simeq R g_{n}^{3}\left(\log \frac{1}{g_{n}}\right) \quad n=1, \cdots, N^{*}
$$

Now since $\frac{g_{n}}{\alpha_{n}} \ll \frac{g_{n-1}}{\alpha_{n-1}} \ll \cdots \ll 1$, it follows that

$$
N \propto \frac{\alpha N^{*}}{g_{N^{*}}}
$$

Solving (5.39), we obtain

$$
\begin{aligned}
& \alpha_{n}=b_{n} R^{1-\frac{1}{2} \cdot 3^{-n+1}} \prod_{K=1}^{n-1}\left(\log \frac{1}{g_{K}}\right)^{\frac{1}{2} \cdot 3^{K-n}}, \\
& g_{n}^{3^{n}}\left(\log \frac{1}{g_{n}}\right)^{3^{n-1}} \prod_{K=1}^{n}\left(\log \frac{1}{g_{K}}\right)^{-\frac{1}{6} \cdot 3^{K}}=R^{-\frac{1}{2}},
\end{aligned}
$$

where we define $\prod_{K=1}^{0} \equiv 1$ and $b_{n}$ is a constant independent of $R$ to be determined later. We note that another constant factor can be included in the right hand side of (5.42), but since it changes the inner layer thickness by a constant factor which can be shown to be of no consequence, can be eliminated there. Using (5.41) - (5.42), we have

$$
\begin{gathered}
\alpha_{n}^{2} \frac{g_{n-1}}{\alpha_{n-1}}=b_{n}^{2} b_{n-1}^{-1} R, \\
\frac{\alpha_{n}}{g_{n}^{3}\left(\log \frac{1}{g_{n}}\right)}=b_{n} R, \frac{g_{n}}{\alpha_{n}}=b_{n}^{-1} R^{-1+3^{-n}} \prod_{K=1}^{n}\left(\log \frac{1}{g_{K}}\right)^{-3^{K-n-1}},
\end{gathered}
$$

where we define $b_{0} \equiv 1$.

Let us now define

$$
K_{N^{*}}=\frac{1-\sum_{1}^{N^{*}}\left(b_{n}<\hat{\theta}_{n}^{\prime 2}>+2 b_{n}^{2} b_{n-1}-1<\widetilde{\theta}_{n}^{2}>\right)}{2 \sum_{1}^{N^{*}} b_{N^{*}}^{-1} \frac{\alpha_{N^{*}}}{g_{N^{*}}} \cdot \frac{g_{n}}{\alpha_{n}}<\left(1-\zeta_{n} \widehat{\theta}_{n}-\widetilde{\theta}_{n+1}^{2}\right)^{2}>}
$$

Then

$$
N_{N^{*}}=K_{N^{*}} R^{1-3^{-N^{*}}} \prod_{K=1}^{N^{*}}\left(\log \frac{1}{g_{K}}\right)^{3^{K-N^{*}-1}}
$$

Fixing $N^{*}$, the $N \max$ can be obtained if we maximize $K_{N^{*}}$. Now from (5.27), (5.33), (5.44), and (5.41) - (5.42), we obtain

$$
\begin{aligned}
& a=2 b_{n+1}^{2} \quad b_{n}^{-2}, \\
& a_{1}=2 K_{N^{*}} b_{N^{*}}^{-2} .
\end{aligned}
$$


Thus

$$
\begin{gathered}
\hat{\theta}_{N^{*}}=\left(\frac{2 K_{N^{*}}}{b_{N^{*}}^{2}}\right)^{\frac{1}{4}} f, \zeta_{N^{*}}=\left(\frac{2 K_{N^{*}}}{b_{N^{*}}^{2}}\right)^{-\frac{1}{4}} \eta . \\
\hat{\theta}_{n}=\left(\frac{2 b_{n+1}}{b_{n}}\right)^{\frac{1}{2}} g, \zeta_{n}=\left(\frac{2 b_{n+1}}{b_{n}}\right)^{-\frac{1}{2}} \eta .
\end{gathered}
$$

Using (5.45) in (5.43) and the fact that $\widetilde{\theta}_{n+1}^{2} \simeq 1-\zeta_{n} \widehat{\theta}_{n}$, we obtain

$K_{N^{*}}=\frac{1-b_{1}^{2} \sum_{1}^{*}-1\left[b_{n}\left(2 b_{n+1} b_{n}^{-1}\right)^{3 / 2}<\left(\frac{d g}{d \eta}\right)^{2}>+\frac{b_{n}}{2}\left(2 b_{n}+1^{b_{n}^{-1}}\right)^{3 / 2}\langle(1-\eta g)>]-b N^{*}\left(2 K^{*} N^{* b} N^{-2}\right)^{3 / 4}<\left(\frac{d f}{d \eta}\right)^{2}>\right.}{2 b^{-1}<(1-\eta f)^{2}>\left(2 K_{N^{*}} b^{-2}\right)^{-1 / 4}}$

Defining:

$$
\int_{0}^{\infty}\left[\left(\frac{d g}{d \eta}\right)^{2}+\frac{1}{2}(1-\eta g)\right] d \eta=3 \beta \quad \int_{0}^{\infty}\left[\left(\frac{d f}{d \eta}\right)^{2}+(1-\eta f)^{2}\right] d \eta=I,
$$

then (5.46) becomes:

$$
2^{3 / 4} I K_{N^{*}}^{3 / 4} b_{N^{*}}^{-1 / 2}=1-6 \beta \sum_{1}^{N^{*}-1}\left(2 b_{n+1}^{3} / b_{n}\right)^{1 / 2}-b_{1}^{2} .
$$

Maximizing $K_{N^{*}}$ with respect to $b_{n}$ gives us the following relations

$$
\begin{gathered}
\sqrt{2} b_{1}=3 \beta b_{2}^{3 / 2} b_{1}^{-3 / 2} \\
\left(b_{n+1}^{3} / b_{n}\right)^{\frac{1}{2}}=3\left(b_{n}^{3} / b_{n-1}\right)^{\frac{1}{2}}, 2 \leq n \leq N^{*}-1 \\
\sqrt{2} 18 \beta b_{N^{*}}^{2} b_{N^{*}-1}^{-1 / 2}=2^{3 / 4} I K_{N^{*}}^{3 / 4}
\end{gathered}
$$

Solving (5.49), we obtain

$$
\begin{gathered}
b_{1}^{2}\left(2.3^{N^{*}}-1\right)=1 \\
b_{n+1}=3^{\frac{1}{2}\left(2 n-3+3^{-n+1}\right)}\left(\frac{\sqrt{2}}{3 \beta}\right)^{1-3^{-n}} b_{1}^{2-3^{-n}} \cdot 1 \leq n \leq N^{*}-1
\end{gathered}
$$

Thus

$$
\begin{aligned}
& K_{N^{*}}=2^{-1 / 3}\left(\frac{1}{I}\right)^{4 / 3}(18 \beta)^{4 / 3} 3^{2 N^{*}-\frac{13}{3}+3^{-N^{*}+1}}\left(\frac{\sqrt{2}}{3 \beta}\right)^{2\left(1-3^{\left.-N^{*}\right)}\right.} b_{1}^{2\left(2-3^{\left.-N^{*}\right)}\right.} \\
& N_{N^{*}}=K_{N^{*}} R^{1-3^{-N^{*}}} \prod_{K=1}^{N^{*}}\left(\log \frac{1}{g_{K}}\right)^{3^{K-N^{*}-1}}
\end{aligned}
$$

Using (5.42), we can rewrite (5.51b) in the following form

$$
\begin{gathered}
N_{N^{*}}=2^{-1 / 3}\left(\frac{1}{I}\right)^{4 / 3}(18 \beta)^{4 / 3} 3^{2 N^{*}}-\frac{13}{3}+3^{-N^{*}+1}\left(\frac{\sqrt{2}}{3 \beta}\right)^{2\left(1-3^{-N^{*}}\right)}\left(2.3^{N^{*}}-1\right)^{-2+3^{-N^{*}}} \\
R^{1-3^{-N^{*}}}(\log R)^{\frac{1}{2}\left(1-3^{-N^{*}}\right)}\left(\frac{1}{2}\right)^{\frac{1}{2}\left(1-3^{-N^{*}}\right)} 3^{-\frac{1}{4}\left(2 N^{*}-1+3^{-N^{*}}\right)}
\end{gathered}
$$

Choosing $N^{*}$ such that $N_{N^{*}} \geq N$, for all $i \neq N^{*}$, keeping $R$ large but fixed and assuming $N^{*}$ will be large and treat it as a continuous variable, we obtain

$$
N^{*}=\frac{1}{\log 3}(\log \log R+\log 2)
$$


and we finally obtain the expression for $N$ as

$$
N \simeq\left(\frac{1}{I}\right)^{4 / 3}\left(\frac{1}{\beta}\right)^{2 / 3}\left(\frac{1}{2}\right) 3^{-41 / 12} R,
$$

where $I=1.062([5])$ and $3 \beta=\frac{20}{9}(6)^{1 / 4}$ (see appendix for more detail of the numerical values of $\beta$ ). The solution (5.54) has the same functional dependence on $R$ as in the non-rotational problem to the leading order terms.

b. Rotation is important $\left[0(1) \ll T_{a} \ll 0(\sqrt{R} \log R)\right]$.

The boundary layer structure in this region is similar to the one discussed in case (a). We consider three regions for each mode, the interior, the intermediate layer, and the inner layer. We define $\frac{\sqrt{T a}}{\alpha n}$ as the thickness of the intermediate layer in the $n$th mode and $\delta_{n}$ as the thickness of the inner layer in the same mode. We also define (5.1) and assume

$$
\alpha_{n} \rightarrow \infty, \delta_{n-1} \ll \frac{\sqrt{T_{a}}}{\alpha_{n-1}} \ll \delta_{n-2} \quad \text { as } R \rightarrow \infty
$$

In the interior of the $\alpha_{n}$ mode, we define (5.3), where $\widetilde{\omega}_{n}, \widetilde{\theta}_{n}, \widetilde{Z}_{n}$ are order one quantities and $\zeta_{n-1}=\frac{z}{\delta_{n-1}}$ is the boundary layer variable for the $(n-1)$ th mode. The basic equations (2.22) - (2.23) then become (5.4) - (5.5) which suggest (5.6) - (5.7). Using (5.55), we obtain (5.10).

In the intermediate layer of the $\alpha_{n}$ mode, we define (5.11), where $\stackrel{\circ}{\omega}_{n}, \stackrel{\circ}{n}_{n}, \stackrel{\mathscr{Z}}{n}_{n}$ are order one quantities and $\xi_{n}=z / \sqrt{T_{a}} / \alpha_{n}$ is the intermediate layer variable for the $n$th mode. Since there is no coupling between the modes in this layer, the equation (2.24) gives (5.12), if we assume (5.13). We also assume that

$$
\frac{1}{\alpha_{n}} \ll \frac{\sqrt{T_{a}}}{\alpha_{n}} \ll 1
$$

The equations (2.22) - (2.23) in this layer become

which suggest that

$$
\begin{gathered}
-\stackrel{\circ}{\omega}_{n}+\frac{1}{B_{n}^{2}} \stackrel{\circ}{n}_{n}+\frac{L_{n} \sqrt{T_{a}}}{\alpha_{n} B_{n}} \frac{d \stackrel{\circ}{Z}_{n}}{d \xi_{n}}=0, \\
\alpha_{n} B_{n} \frac{d \stackrel{\circ}{\omega}_{n}}{d \xi_{n}}=\sqrt{T_{a}} L_{n} \stackrel{\circ}{Z}_{n}
\end{gathered}
$$

$$
L_{n}=\alpha_{n} B_{n} / \sqrt{T_{a}}
$$

Using (5.58) in (5.57), we obtain

$$
\begin{aligned}
& -\stackrel{\circ}{\omega}_{n}+\stackrel{\circ}{n}_{n}+\frac{d \stackrel{\circ}{n}_{n}}{d \xi_{n}}=0, \frac{d \stackrel{\circ}{n}_{n}}{d \xi_{n}}=\stackrel{\check{Z}}{n}_{n}, \\
& B_{n}=1 \text {. }
\end{aligned}
$$

The form of the solutions to (5.12) and (5.59a) as $\xi_{n} \rightarrow 0$ can be found, after imposing the requirement that $\omega_{n} \rightarrow 0$, to be (5.20). The appropriate expression for $1-\sum_{n} \omega_{n} \theta_{n}$ is

$$
1-\sum_{n} \omega_{n} \theta_{n}=\left\{\begin{array}{l}
0 \text { for } z \simeq 0\left(\sqrt{T_{a}} / \alpha_{n}\right) \\
1-\widehat{\omega}_{n}\left(\zeta_{n}\right) \widehat{\theta}_{n}\left(\zeta_{n}\right)-\widetilde{\omega}_{n+1}\left(\zeta_{n}\right) \widetilde{\theta}_{n+1}\left(\zeta_{n}\right) \text { for } z \simeq 0\left(\delta_{n}\right)
\end{array}\right.
$$

where $\widehat{\omega}_{n}, \widehat{\theta}_{n}$ are the solutions in the inner layer.

In the inner layer of the $\alpha_{n}$ mode, we define (5.22), where $\widehat{\omega}_{n}, \widehat{\theta}_{n}, \widehat{Z}_{n}$ are order one quantities and $\zeta_{n}=z / \delta_{n}$ is the inner layer variable of the $n$th mode. Matching conditions yield 


$$
\begin{gathered}
C_{n}=\sqrt{\frac{2}{T_{a}}} \alpha_{n} \delta_{n}\left(\log \frac{\sqrt{T_{a}}}{\alpha_{n} \delta_{n}}\right)^{\frac{1}{2}}, \\
M_{n}=\sqrt{\frac{2}{T_{a}}} \alpha_{n}\left(\log \frac{\sqrt{T_{a}}}{\alpha_{n} \delta_{n}}\right)^{\frac{1}{2}}, \\
\widehat{\omega}_{n}\left(\zeta_{n} \rightarrow \infty\right) \simeq \zeta_{n}, \widehat{\theta}_{n}\left(\zeta_{n} \rightarrow \infty\right) \simeq \frac{1}{\zeta_{n}}, \quad \widehat{Z}_{n}\left(\zeta_{n} \rightarrow \infty\right) \simeq 1 .
\end{gathered}
$$

The governing equations $(2.22)-(2.24)$ then reduce to

$$
\begin{gathered}
\frac{d \widehat{Z}_{n}}{d \zeta_{n}}=0, \frac{d \widehat{\omega}_{n}}{d \zeta_{n}}=\widehat{Z}_{n}, \\
T_{a} \frac{d^{2} \widehat{\theta}_{n}}{d \zeta_{n}}+2 N R \alpha_{n}^{2} \delta_{n}^{2}\left(\log \frac{\sqrt{T_{a}}}{\alpha_{n} \delta_{n}}\right)\left(1-\widehat{\omega}_{n} \widehat{\theta}_{n}-\widetilde{\omega}_{n+1} \widetilde{\theta}_{n+1}\right) \widehat{\omega}_{n}=0
\end{gathered}
$$

In this layer, one should also consider the functions $\widetilde{\omega}_{n+1}, \widetilde{\theta}_{n+1}$ and we have

$$
\begin{gathered}
-\alpha_{n+1}^{2} \widetilde{\theta}_{n+1}+N R\left(1-\widehat{\omega}_{n} \widehat{\theta}_{n}-\widetilde{\omega}_{n+1} \widetilde{\theta}_{n+1}\right) \widetilde{\omega}_{n+1}=0 \\
\frac{d \widetilde{\omega}_{n+1}}{d \zeta_{n}}=\widetilde{Z}_{n+1}, \widetilde{\omega}_{n}=\widetilde{\theta}_{n} .
\end{gathered}
$$

Assuming the total number of the modes in $N^{*}$, then for $n \neq N^{*}, \widetilde{\theta}_{n+1} \neq 0$ and $(5.61 \mathrm{~b})-(5.62 \mathrm{a})$ then give

which suggests that

$$
T_{a} \frac{d^{2} \widehat{\theta}_{n}}{d \zeta_{n}^{2}}+2 \alpha_{n}^{2} \alpha_{n+1}^{2} \delta_{n}^{4}\left(\log \frac{\sqrt{T_{a}}}{\alpha_{n} \delta_{n}}\right) \widehat{\omega}_{n}=0
$$

$$
2 \alpha_{n}^{2} \delta_{n}^{4}\left(\log \frac{\sqrt{T_{a}}}{\alpha_{n} \delta_{n}}\right) \alpha_{n+1}^{2}=a T_{a}
$$

where $a=0(1)$ is a constant and will be determined later. Thus (5.63) reduces to a form like (5.28) satisfying the boundary condition (5.29). For $n \neq N^{*}$ then as $\widetilde{\theta}_{n+1}$ decreases to zero, $\widehat{\theta}_{n}$ must merge with $1-\widehat{\omega}_{n} \widehat{\theta}_{n}=0$ which is the leading order term of $(5.61 \mathrm{~b})$. Now (5.61a) gives (5.30) and using the transformation $\hat{\theta}_{n}=a^{1 / 4} g, \zeta_{n}=a^{-1 / 4} \eta$ and (5.30) in (5.28), we obtain (5.31) - (5.32). For $n=N^{*}, \widetilde{\theta}_{n+1} \equiv 0$ and $(5.62 \mathrm{a}, \mathrm{b})$ always satisfied. $(5.61 \mathrm{~b})$ then suggests that

$$
2 N R \alpha_{N^{*}}^{2} \delta_{N^{*}}^{4}\left(\log \frac{\sqrt{T_{a}}}{\alpha_{N^{*}} \delta_{N^{*}}}\right)=a_{1} T_{a},
$$

where $a_{1}=0(1)$ is a constant and will be determined later. Thus (5.61b) reduces to a form like (5.34) satisfying (5.35). Using the transformation $\xi_{N^{*}}=a_{n}^{-1 / 4} \eta, \theta_{N^{*}}=a_{1}^{1 / 4} f$ in (5.34) - (5.35), we get (5.36).

To evaluate the expression (2.11), we write the expressions $\left\langle|\nabla \theta|^{2}\right\rangle$ and $\left\langle(1-\overline{\omega \theta})^{2}\right\rangle$ to the leading order terms as:

$$
\begin{gathered}
<|\nabla \theta|^{2}>\simeq \sum_{1}^{N^{*}}\left[2 \alpha_{n}^{2} \frac{g_{n-1}}{\alpha_{n-1}}<\widetilde{\theta}_{n}^{2}>+\frac{T_{a} \alpha_{n}}{g_{n}^{3}\left(\log \frac{\sqrt{T_{a}}}{g_{n}}\right)}<\widehat{\theta}_{n}^{\prime 2}>\right] \\
<(1-\overline{\omega \theta})^{2}>\simeq 2 \sum_{1}^{N^{*}} \frac{g_{n}}{\alpha_{n}}<\left(1-\zeta_{n} \hat{\theta}_{n}-\widetilde{\theta}_{n+1}^{2}\right)^{2}>
\end{gathered}
$$

where we define

$$
\delta_{n}=\frac{g_{n}}{\alpha_{n}},<\widetilde{\theta}_{n}^{2}>=\frac{1}{2}, \widetilde{\theta}_{N^{*}+1} \equiv 0 .
$$

Using these expressions in (2.11), we have 


$$
N \simeq \frac{1-\frac{1}{R} \sum_{1}^{N^{*}}\left[2 \alpha_{n}^{2} \frac{g_{n-1}}{\alpha_{n-1}}<\widetilde{\theta}_{n}^{2}>+\frac{T_{a} \alpha_{n}}{g_{n}^{3}\left(\log \sqrt{T a} / g_{n}\right)}<\widehat{\theta}_{n}^{\prime 2}>\right]}{2 \sum_{1}^{N^{*}} \frac{\alpha_{N^{*}}}{g_{N^{*}}} \cdot \frac{g_{n}}{\alpha_{n}}<\left(1-\zeta_{n} \widehat{\theta}_{n}-\widetilde{\theta}_{n+1}^{2}\right)^{2}>} \cdot \frac{\alpha_{N^{*}}}{b_{N^{*}}} .
$$

To determine $\alpha_{n}$ and $g_{n}$, we again require

$$
\alpha_{n}^{2} g_{n-1} \simeq R \alpha_{n-1}, \alpha_{n} T_{a} \simeq R g_{n}^{3}\left(\log \frac{\sqrt{T_{a}}}{g_{n}}\right) n=1, \cdots, N^{*}
$$

Now since $\frac{g_{n}}{\alpha_{n}} \ll \frac{g_{n-1}}{\alpha_{n-1}} \ll \cdots \ll 1$, we obtain

$$
N \propto \frac{\alpha N^{*}}{g_{N^{*}}}
$$

Solving (5.67), we obtain

$$
\begin{gathered}
a_{n}=b_{n} R^{1-\frac{1}{2} 3^{-n+1}} T_{a}^{-\frac{1}{4}\left(1-3^{-n+1}\right)} \prod_{K=1}^{n}\left(\log \frac{\sqrt{T_{a}}}{g_{K}}\right)^{\frac{1}{2} \cdot 3^{K-n}} \\
g_{n}^{3^{n}}\left(\log \frac{\sqrt{T_{n}}}{g_{n}}\right)^{3^{n-1}} \prod_{K=1}^{n}\left(\log \frac{\sqrt{T_{a}}}{g_{K}}\right)^{-\frac{1}{6} \cdot 3^{K}}=T_{a}^{\frac{1}{4}\left(1+3^{n}\right)} R^{-\frac{1}{2}}
\end{gathered}
$$

where again we define $\prod_{\kappa=1}^{0} \equiv 1$ and $b_{n}$ is a constant independent of $R$ and $T_{\mathrm{a}}$. Using $(5.69 \mathrm{a}, \mathrm{b})$, we have

$$
\begin{gathered}
a_{n}^{2} g_{n-1}=\alpha_{n-1} b_{n}^{2} b_{n-1}^{-1} R \\
\alpha_{n} T_{a}=b_{n} g_{n}^{3}\left(\log \frac{\sqrt{T_{a}}}{g_{n}}\right) R \\
g_{n}=b_{n}^{-1} \alpha_{n} R^{-1+3^{-n}} T_{a}^{\frac{1}{2}\left(1-3^{-n}\right)} \prod_{K=1}^{n}\left(\log \frac{\sqrt{T_{a}}}{g_{K}}\right)^{-3^{K-n-1}},
\end{gathered}
$$

where we define $b_{0} \equiv 1$.

Now using (5.55) and $(5.69 \mathrm{a}, \mathrm{b})$, we find that for sufficiently small $\left|T_{a}-0(1)\right|$ we now have infinitely many modes. However as $T_{a}$ increases in the range $0(1) \ll T_{a} \ll 0(\sqrt{R} \log R)$, the number of modes decreases. In particular, for $0\left(R^{1 / 5}\right) \ll T_{a} \ll 0(\sqrt{R} \log R)$ we have only two modes namely $\alpha_{1}$ and $\alpha_{2}$. Let us now define

$$
K_{N^{*}}=\frac{1-\sum_{1}^{N^{*}}\left(b_{n}<\hat{\theta}_{n}^{\prime 2}>+2 b_{n}^{2} b_{n-1}^{-1}<\widetilde{\theta}_{n}^{2}>\right)}{2 \sum_{1}^{N^{*}} b_{N^{*}}^{-1} \frac{\alpha_{N^{*}}}{g_{N^{*}}} \frac{g_{n}}{\alpha_{n}}<\left(1-\zeta_{n} \widehat{\theta}_{n}-\widetilde{\theta}_{n+1}^{2}\right)^{2}>}
$$

where $N^{*}$ is the total number of modes and can be easily determined for a given $T_{a}$ in the range $0(1) \ll T_{a} \ll 0(\sqrt{R} \log R)$, by the inequality

$$
\frac{\sqrt{T_{a}}}{\alpha_{N^{*}}} \ll \frac{g_{N^{*}-1}}{\alpha_{N^{*}-1}}
$$

Then

$$
N_{N^{*}}=K_{N^{*}} R^{1-3^{-N^{*}}} T_{a}^{-\frac{1}{2}\left(1-3^{-N^{*}}\right)} \prod_{K=1}^{N^{*}}\left(\log \frac{\sqrt{T_{a}}}{g_{K}}\right)^{3^{K-N^{*}-1}}
$$

Now from (5.64) - (5.65), (5.69a,b), and (5.72), we get the relations (5.45). Using these relations into (5.70) we can easily obtain the expressions (5.46) - (5.51a). Using (5.69b), we can rewrite 
(5.72) in the following form:

$$
\begin{gathered}
N_{N^{*}}=2^{-\frac{1}{3}} I^{-\frac{4}{3}}(18 \beta)^{\frac{4}{3}} 3^{2 N^{*}-\frac{13}{3}+3^{-N^{*}+1}}\left(\frac{\sqrt{2}}{3 \beta}\right)^{2\left(1-3^{-N^{*}}\right)}\left(2 \cdot 3^{N^{*}}-1\right)^{-2+3^{-N^{*}}} \\
R^{1-3-N^{*}} T_{a}^{-\frac{1}{2}\left(1-3^{-N^{*}}\right)} \prod_{K^{*}=1}^{N^{*}}\left[\log \frac{R^{\frac{1}{2} \cdot 3^{-K}}}{T_{a}^{-\frac{1}{4}\left(1-3^{-K}\right)}}\right]^{K-N^{*}-1}
\end{gathered}
$$

c. Strongly rotating system $\left[0(\sqrt{R} \log R) \ll T_{a} \ll 0(R)\right]$.

From the preceding section, it is clear that as $T_{a} \rightarrow 0(\sqrt{R} \log R)$, the number of the preferred modes approaches one and the Nusselt number has the functional dependence $\left(\frac{R^{2} \log R}{T_{a}}\right)^{1 / 3}$ It is then expected that in the case of strongly rotating system, we have only one mode, at least, for the limiting case of $T_{a} \rightarrow 0(\sqrt{R} \log R)$. Single wave number analysis (section 4.e) clearly has shown that the inner layer thickness is independent of the wave number to the leading order terms. Thus there is no hope to decrease the order of this thin layer by allowing more modes. Therefore there is not a multiboundary layer structure for this case that exists.

\section{APPENDIX}

We compute $\beta$ by solving

$$
\frac{d^{2} g}{d \eta^{2}}+\eta=0
$$

Satisfying $g(0)=0$ and $g=\frac{1}{\eta}$ as $\eta \rightarrow \infty$

(A-1) and boundary condition $g(0)=0$ give

$$
g(\eta)=A \eta-\frac{1}{6} \eta^{3}
$$

We must now find a value $\eta^{*}$ at which (A-3) and the boundary conditions for $\eta \rightarrow \infty$, together with their first derivatives, are continuous, i.e.,

Solving then, we obtain $\eta^{*}=(6)^{\frac{1}{4}}$. Thus

$$
\begin{gathered}
A \eta^{*}-\frac{1}{6} \eta^{* 3}=\frac{1}{\eta^{*}} \\
A-\frac{1}{2} \eta^{* 2}=-\eta^{*^{-2}} .
\end{gathered}
$$

and so

$$
\begin{array}{ll}
g(\eta)=\frac{\sqrt{6}}{3} \eta-\frac{1}{6} \eta^{3} & \text { for } 0 \leq \eta \leq(6)^{\frac{1}{4}} \\
g(\eta)=\frac{1}{\eta} & \text { for } \quad \eta \geq(6)^{\frac{1}{4}}
\end{array}
$$

$$
3 \beta=\int_{0}^{\infty}\left[\left(\frac{d g}{d \eta}\right)^{2}+\frac{1}{2}(1-\eta g)\right] d \eta=\frac{20}{9}(6)^{\frac{1}{4}}
$$




\section{REFERENCES}

1. BUSSE, F.H., \& JOSEPH, D.D., Bounds for heat transport in a porous layer, Journal of Fluid Mechanics, 54 (1972), 521-543.

2. CHAN, S.K., Infinite Prandtl number turbulent convection. Studies in Applied Mathematics 50 (1971), 13-49.

3. CHAN, S.K., Investigation of turbulent convection under rotational constraint, Journal of Fluid Mechanics 64 (1974), 477-506.

4. CHANDRASEKHAR, S., Hydrodynamics and Hydromagnetic Stability, Oxford University Press, 1961.

5. GUPTA, V.P. \& JOSEPH, D.D., Bounds for heat transport in a porous layer, Journal of Fluid Mechanics 57 (1973), 491-514.

6. HOWARD, L.N., Heat transport by turbulent convection, Journal of Fluid Mechanics 17 (1963), 405-432.

7. LAPWOOD, E.R., Convection of a fluid in a porous medium, Proceedings of Cambridge Philosophical Society 44 (1948), 508-521.

8. MALKUS, W.V.R., The heat transport and spectrum of thermal turbulence, Proceedings of Royal Society 255A (1954), 196-212. 


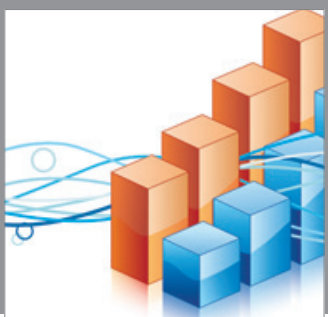

Advances in

Operations Research

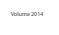

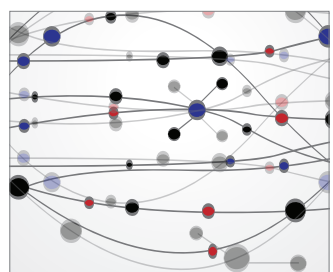

\section{The Scientific} World Journal
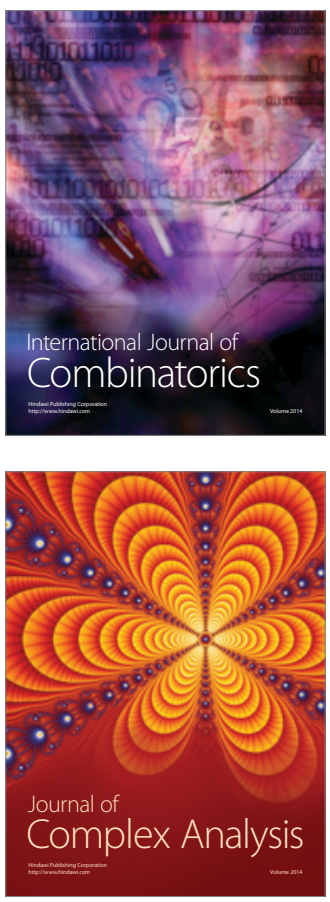

International Journal of

Mathematics and

Mathematical

Sciences
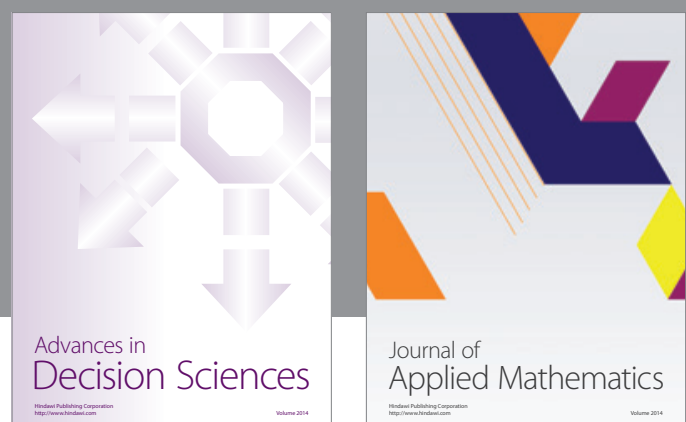

Journal of

Applied Mathematics
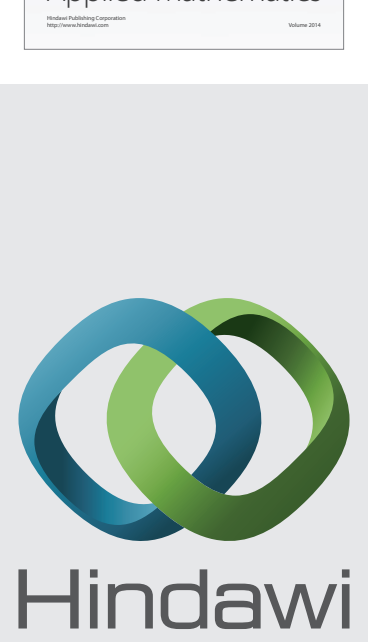

Submit your manuscripts at http://www.hindawi.com
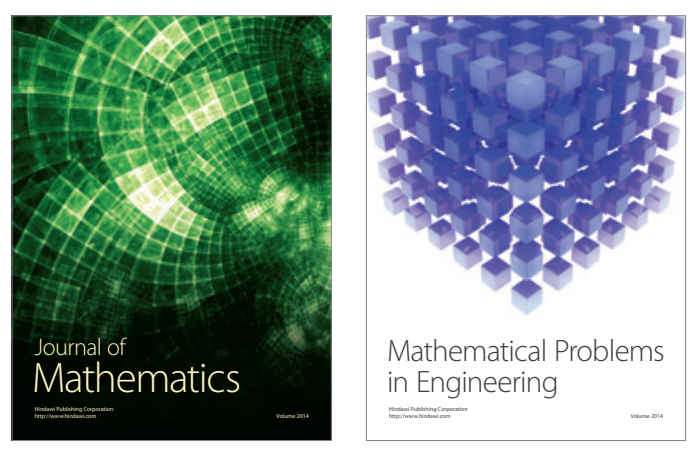

Mathematical Problems in Engineering
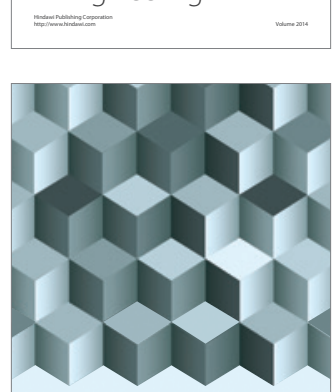

Journal of

Function Spaces
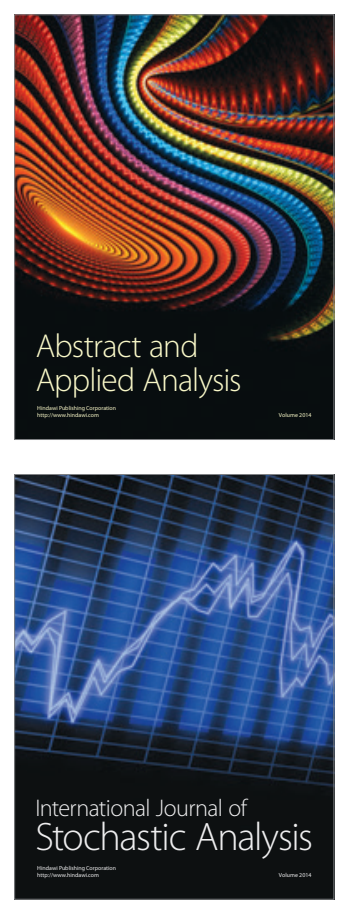

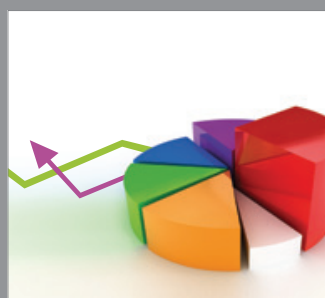

ournal of

Probability and Statistics

Promensencen
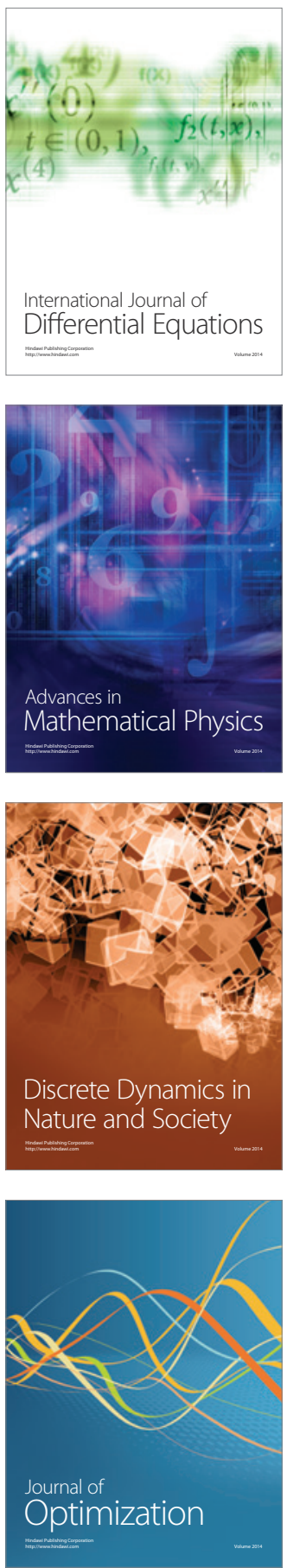